\title{
Mann-Type Viscosity Approximation Methods for Multivalued Variational Inclusions with Finitely Many Variational Inequality Constraints in Banach Spaces
}

\author{
Lu-Chuan Ceng, ${ }^{1}$ Abdul Latif, ${ }^{2}$ and Abdullah E. Al-Mazrooei ${ }^{3}$ \\ ${ }^{1}$ Department of Mathematics, Shanghai Normal University, Scientific Computing Key Laboratory of Shanghai Universities, \\ Shanghai 200234, China \\ ${ }^{2}$ Department of Mathematics, Faculty of Science, King Abdulaziz University, P.O. Box 80203, Jeddah 21589, Saudi Arabia \\ ${ }^{3}$ Department of Mathematics, King Abdulaziz University, P.O. Box 80203, Jeddah 21589, Saudi Arabia
}

Correspondence should be addressed to Abdul Latif; alatif@kau.edu.sa

Received 13 September 2013; Accepted 23 October 2013

Academic Editor: Chi-Ming Chen

Copyright (c) 2013 Lu-Chuan Ceng et al. This is an open access article distributed under the Creative Commons Attribution License, which permits unrestricted use, distribution, and reproduction in any medium, provided the original work is properly cited.

\begin{abstract}
We introduce Mann-type viscosity approximation methods for finding solutions of a multivalued variational inclusion (MVVI) which are also common ones of finitely many variational inequality problems and common fixed points of a countable family of nonexpansive mappings in real smooth Banach spaces. Here the Mann-type viscosity approximation methods are based on the Mann iteration method and viscosity approximation method. We consider and analyze Mann-type viscosity iterative algorithms not only in the setting of uniformly convex and 2-uniformly smooth Banach space but also in a uniformly convex Banach space having a uniformly Gáteaux differentiable norm. Under suitable assumptions, we derive some strong convergence theorems. In addition, we also give some applications of these theorems; for instance, we prove strong convergence theorems for finding a common fixed point of a finite family of strictly pseudocontractive mappings and a countable family of nonexpansive mappings in uniformly convex and 2-uniformly smooth Banach spaces. The results presented in this paper improve, extend, supplement, and develop the corresponding results announced in the earlier and very recent literature.
\end{abstract}

\section{Introduction}

Let $X$ be a real Banach space whose dual space is denoted by $X^{*}$. The normalized duality mapping $J: X \rightarrow 2^{X^{*}}$ is defined by

$$
J(x)=\left\{x^{*} \in X^{*}:\left\langle x, x^{*}\right\rangle=\|x\|^{2}=\left\|x^{*}\right\|^{2}\right\}, \quad \forall x \in X,
$$

where $\langle\cdot, \cdot\rangle$ denotes the generalized duality pairing. It is an immediate consequence of the Hahn-Banach theorem that $J(x)$ is nonempty for each $x \in X$. Let $U=\{x \in X:\|x\|=1\}$ denote the unite sphere of $X$. A Banach space $X$ is said to be uniformly convex if, for each $\epsilon \in(0,2]$, there exists $\delta>0$ such that, for all $x, y \in U$,

$$
\|x-y\| \geq \epsilon \Longrightarrow \frac{\|x+y\|}{2} \leq 1-\delta .
$$

It is known that a uniformly convex Banach space is reflexive and strict convex. A Banach space $X$ is said to be smooth if the limit

$$
\lim _{t \rightarrow 0} \frac{\|x+t y\|-\|x\|}{t}
$$

exists for all $x, y \in U$; in this case, $X$ is also said to have a Gáteaux differentiable norm. $X$ is said to have a uniformly Gáteaux differentiable norm if, for each $y \in$ $U$, the limit is attained uniformly for $x \in U$. Moreover, it is said to be uniformly smooth if this limit is attained uniformly for $x, y \in U$. The norm of $X$ is said to be the Fréchet differential if, for each $x \in U$, this limit is attained uniformly for $y \in U$. In addition, we define a function $\rho$ : 
$[0, \infty) \rightarrow[0, \infty)$ called the modulus of smoothness of $X$ as follows:

$$
\begin{aligned}
\rho(\tau)=\sup \left\{\frac{1}{2}(\|x+y\|+\|x-y\|)\right. \\
-1: x, y \in X,\|x\|=1,\|y\|=\tau\} .
\end{aligned}
$$

It is known that $X$ is uniformly smooth if and only if $\lim _{\tau \rightarrow 0} \rho(\tau) / \tau=0$. Let $q$ be a fixed real number with $1<q \leq$ 2. Then a Banach space $X$ is said to be $q$-uniformly smooth if there exists a constant $c>0$ such that $\rho(\tau) \leq c \tau^{q}$ for all $\tau>0$. It is well-known that no Banach space is $q$-uniformly smooth for $q>2$. In addition, it is also known that $J$ is single-valued if and only if $X$ is smooth, whereas if $X$ is uniformly smooth, then the mapping $J$ is norm-to-norm uniformly continuous on bounded subsets of $X$. If $X$ has a uniformly Gáteaux differentiable norm then the duality mapping $J$ is norm-toweak $^{*}$ uniformly continuous on bounded subsets of $X$.

Let $C$ be a nonempty closed convex subset of a real Banach space $X$. A mapping $T: C \rightarrow C$ is called nonexpansive if

$$
\|T x-T y\| \leq\|x-y\|, \quad \forall x, y \in C .
$$

The set of fixed points of $T$ is denoted by $\operatorname{Fix}(T)$. We use the notation $\rightarrow$ to indicate the weak convergence and the one $\rightarrow$ to indicate the strong convergence.

Definition 1. Let $A: C \rightarrow X$ be a mapping of $C$ into $X$. Then $A$ is said to be

(i) accretive if for each $x, y \in C$ there exists $j(x-y) \in$ $J(x-y)$ such that

$$
\langle A x-A y, j(x-y)\rangle \geq 0
$$

where $J$ is the normalized duality mapping;

(ii) $\alpha$-strongly accretive if for each $x, y \in C$ there exists $j(x-y) \in J(x-y)$ such that

$$
\langle A x-A y, j(x-y)\rangle \geq \alpha\|x-y\|^{2}
$$

for some $\alpha \in(0,1)$;

(iii) $\beta$-inverse strongly accretive if for each $x, y \in C$ there exists $j(x-y) \in J(x-y)$ such that

$$
\langle A x-A y, j(x-y)\rangle \geq \beta\|A x-A y\|^{2}
$$

for some $\beta>0$;

(iv) $\lambda$-strictly pseudocontractive if for each $x, y \in C$ there exists $j(x-y) \in J(x-y)$ such that

$$
\langle A x-A y, j(x-y)\rangle \leq\|x-y\|^{2}-\lambda\|x-y-(A x-A y)\|^{2}
$$

for some $\lambda \in(0,1)$.
Let $X$ be a real smooth Banach space. Let $C$ be a nonempty closed convex subset of $X$ and let $A: C \rightarrow X$ be a nonlinear mapping. The so-called variational inequality problem (VIP) is the problem of finding $x^{*} \in C$ such that

$$
\left\langle A x^{*}, J\left(x-x^{*}\right)\right\rangle \geq 0, \forall x \in C
$$

which was considered by Aoyama et al. [1]. Note that VIP (10) is connected with the fixed point problem for nonlinear mapping (see e.g., [2]), the problem of finding a zero point of a nonlinear operator (see e.g., [3]), and so on. In particular, whenever $X=H$ a Hilbert space, the VIP (10) reduces to the classical VIP of finding $x^{*} \in C$ such that

$$
\left\langle A x^{*}, x-x^{*}\right\rangle \geq 0, \quad \forall x \in C,
$$

whose solution set is denoted by $\operatorname{VI}(C, A)$. Recently, in order to find a solution of VIP (10), Aoyama et al. [1] introduced Mann-type iterative scheme for an accretive operator $A$ as follows:

$$
x_{n+1}=\alpha_{n} x_{n}+\left(1-\alpha_{n}\right) \Pi_{C}\left(x_{n}-\lambda_{n} A x_{n}\right), \quad \forall n \geq 1,
$$

where $\Pi_{C}$ is a sunny nonexpansive retraction from $X$ onto $C$. Then they proved a weak convergence theorem.

Definition 2. Let $C$ be a nonempty convex subset of a real Banach space $X$. Let $\left\{T_{i}\right\}_{i=1}^{N}$ be a finite family of nonexpansive mappings of $C$ into itself and let $\lambda_{1}, \ldots, \lambda_{N}$ be real numbers such that $0 \leq \lambda_{i} \leq 1$ for every $i=1, \ldots, N$. Define a mapping $K: C \rightarrow C$ as follows:

$$
\begin{gathered}
U_{1}=\lambda_{1} T_{1}+\left(1-\lambda_{1}\right) I \\
U_{2}=\lambda_{2} T_{2} U_{1}+\left(1-\lambda_{2}\right) U_{1} \\
U_{3}=\lambda_{3} T_{3} U_{2}+\left(1-\lambda_{3}\right) U_{2} \\
\vdots \\
U_{N-1}=\lambda_{N-1} T_{N-1} U_{N-2}+\left(1-\lambda_{N-1}\right) U_{N-2} \\
K=U_{N}=\lambda_{N} T_{N} U_{N-1}+\left(1-\lambda_{N}\right) U_{N-1} .
\end{gathered}
$$

Such a mapping $K$ is called the $K$-mapping generated by $T_{1}, \ldots, T_{N}$ and $\lambda_{1}, \ldots, \lambda_{N}$.

Lemma 3 (see [4]). Let $C$ be a nonempty closed convex subset of a strictly convex Banach space. Let $\left\{T_{i}\right\}_{i=1}^{N}$ be a finite family of nonexpansive mappings of $C$ into itself with $\cap_{i=1}^{N} \operatorname{Fix}\left(T_{i}\right) \neq \emptyset$ and let $\lambda_{1}, \ldots, \lambda_{N}$ be real numbers such that $0<\lambda_{i}<1$ for every $i=1, \ldots, N-1$ and $0<\lambda_{N} \leq 1$. Let $K$ be the $K$-mapping generated by $T_{1}, \ldots, T_{N}$ and $\lambda_{1}, \ldots, \lambda_{N}$. Then $\operatorname{Fix}(K)=\cap_{i=1}^{N} \operatorname{Fix}\left(T_{i}\right)$.

From Lemma 3, it is easy to see that the $K$-mapping is a nonexpansive mapping.

On the other hand, let $C B(X)$ be the family of all nonempty, closed, and bounded subsets of a real smooth 
Banach space $X$. Also, we denote by $H(\cdot, \cdot)$ the Hausdorff metric on $C B(X)$ defined by

$$
\begin{array}{r}
H(A, B):=\max \left\{\sup _{x \in B} \inf _{y \in A} d(x, y), \sup _{x \in A} \inf _{y \in B} d(x, y)\right\}, \\
\forall A, B \in C B(X) .
\end{array}
$$

Let $T, F: X \rightarrow C B(X)$ be two multivalued mappings, let $A$ : $D(A) \subset X \rightarrow 2^{X}$ be an $m$-accretive mapping, let $g: X \rightarrow$ $D(A)$ be a single-valued mapping, and let $N(\cdot, \cdot): X \times X \rightarrow X$ be a nonlinear mapping. Then for any given $v \in X, \lambda>0$, Chidume et al. [5] introduced and studied the multivalued variational inclusion (MVVI) of finding $x \in D(A)$ such that $(x, w, k)$ is a solution of the following:

$$
v \in N(w, k)+\lambda A(g(x)), \quad \forall w \in T x, k \in F x .
$$

If $v=0$ and $\lambda=1$, then the MVVI (15) reduces to the problem of finding $x \in D(A)$ such that $(x, w, k)$ is a solution of the following:

$$
0 \in N(w, k)+A(g(x)), \quad \forall w \in T x, k \in F x .
$$

We denote by $\Gamma$ the set of such solutions $x$ for MVVI (16).

The authors [5] established an existence theorem for MVVI (15) in a smooth Banach space $X$ and then proved that the sequence generated by their iterative algorithm converges strongly to a solution of MVVI (16).

Theorem 4 (see [5, Theorem 3.2]). Let $X$ be a real smooth Banach space. Let T, F: $X \rightarrow C B(X)$, and $A: D(A) \subset$ $X \rightarrow 2^{X}$ be three multivalued mappings, let $g: X \rightarrow D(A)$ be a single-valued mapping, and let $N(\cdot, \cdot): X \times X \rightarrow X$ be a single-valued continuous mapping satisfying the following conditions:

(C1) $A \circ g: X \rightarrow 2^{X}$ is $m$-accretive and $H$-uniformly continuous;

(C2) $T: X \rightarrow C B(X)$ is H-uniformly continuous;

(C3) $F: X \rightarrow C B(X)$ is H-uniformly continuous;

(C4) the mapping $x \mapsto N(x, y)$ is $\phi$-strongly accretive and $\mu$-H-Lipschitz with respect to the mapping $T$, where $\phi$ : $[0, \infty) \rightarrow[0, \infty)$ is a strictly increasing function with $\phi(0)=0$;

(C5) the mapping $y \mapsto N(x, y)$ is accretive and $\xi-H$ Lipschitz with respect to the mapping $F$.

For arbitrary $x_{0} \in D(A)$, define the sequence $\left\{x_{n}\right\}$ iteratively by

$$
x_{n+1}=x_{n}-\sigma_{n}\left(N\left(w_{n}, k_{n}\right)+u_{n}\right), u_{n} \in A\left(g\left(x_{n}\right)\right),
$$

where $\left\{u_{n}\right\}$ is defined by

$$
\begin{aligned}
& \left\|u_{n}-u_{n+1}\right\| \\
& \quad \leq(1+\varepsilon) H\left(A\left(g\left(x_{n+1}\right)\right), A\left(g\left(x_{n}\right)\right)\right), \forall n \geq 0,
\end{aligned}
$$

for any $w_{n} \in T x_{n}, k_{n} \in F x_{n}$, and some $\varepsilon>0$, where $\left\{\sigma_{n}\right\}$ is a positive real sequence such that $\lim _{n \rightarrow \infty} \sigma_{n}=0, \sum_{n=0}^{\infty} \sigma_{n}=\infty$.
Then, there exists $\bar{d}>0$ such that, for $0<\sigma_{n} \leq \bar{d}$ and for all $n \geq 0,\left\{x_{n}\right\}$ converges strongly to $\bar{x} \in \Gamma$, and, for any $w \in T \bar{x}$ and $k \in F \bar{x},(\bar{x}, w, k)$ is a solution of the MVVI (16).

Let $C$ be a nonempty closed convex subset of a real smooth Banach space $X$ and let $\Pi_{C}$ be a sunny nonexpansive retraction from $X$ onto $C$. Let $f: C \rightarrow C$ be a contraction with coefficient $\rho \in(0,1)$. Motivated and inspired by the research going on this area, we introduce Mann-type viscosity approximation methods for finding solutions of the MVVI (16) which are also common ones of finitely many variational inequality problems and common fixed points of a countable family of nonexpansive mappings. Here, the Mann-type viscosity approximation methods are based on the Mann iteration method and viscosity approximation method. We consider and analyze Mann-type viscosity iterative algorithms not only in the setting of uniformly convex and 2-uniformly smooth Banach space but also in a uniformly convex Banach space having a uniformly Gáteaux differentiable norm. Under suitable assumptions, we derive some strong convergence theorems. In addition, we also give some applications of these theorems; for instance, we prove strong convergence theorems for finding a common fixed point of a finite family of $\eta_{i}$-strictly pseudocontractive mappings $(i=1, \ldots, N)$ and a countable family of nonexpansive mappings in uniformly convex and 2-uniformly smooth Banach spaces. The results presented in this paper improve, extend, supplement, and develop the corresponding results announced in the earlier and very recent literature; see, for example, [6-11].

\section{Preliminaries}

Let $X$ be a real Banach space with dual $X^{*}$. We denote by $J$ the normalized duality mapping from $X$ to $2^{X^{*}}$ defined by

$$
J(x)=\left\{x^{*} \in X^{*}:\left\langle x, x^{*}\right\rangle=\|x\|^{2}=\left\|x^{*}\right\|^{2}\right\},
$$

where $\langle\cdot, \cdot\rangle$ denotes the generalized duality pairing. Throughout this paper, the single-valued normalized duality map is still denoted by $J$. Unless otherwise stated, we assume that $X$ is a smooth Banach space with dual $X^{*}$.

A multivalued mapping $A: D(A) \subseteq X \rightarrow 2^{X}$ is said to be

(i) accretive, if

$$
\langle u-v, J(x-y)\rangle \geq 0, \quad \forall u \in A x, v \in A y ;
$$

(ii) $m$-accretive, if $A$ is accretive and $(I+r A)(D(A))=X$, for all $r>0$, where $I$ is the identity mapping;

(iii) $\zeta$-inverse strongly accretive, if there exists a constant $\zeta>0$ such that

$\langle u-v, J(x-y)\rangle \geq \zeta\|u-v\|^{2}, \quad \forall u \in A x, v \in A y ;$

(iv) $\phi$-strongly accretive, if there exists a strictly increasing continuous function $\phi:[0, \infty) \rightarrow[0, \infty)$ with $\phi(0)=0$ such that

$$
\begin{aligned}
& \langle u-v, J(x-y)\rangle, \\
& \quad \geq \phi(\|x-y\|)\|x-y\|, \quad \forall u \in A x, v \in A y ;
\end{aligned}
$$


(v) $\phi$-expansive, if

$$
\|u-v\| \geq \phi(\|x-y\|), \quad \forall u \in A x, \quad v \in A y .
$$

It is easy to see that if $A$ is $\phi$-strongly accretive, then $A$ is $\phi$-expansive.

A mapping $T: X \rightarrow C B(X)$ is said to be $H$-uniformly continuous, if for any given $\varepsilon>0$, there exists a $\delta>0$ such that whenever $\|x-y\|<\delta$ then $H(T x, T y)<\varepsilon$.

A mapping $N: X \times X \rightarrow X$ is $\phi$-strongly accretive, with respect to $T: X \rightarrow C B(X)$, in the first argument if

$$
\begin{array}{r}
\langle N(u, z)-N(v, z), J(x-y)\rangle \geq \phi(\|x-y\|)\|x-y\|, \\
\forall u \in T x, v \in T y .
\end{array}
$$

A mapping $S: X \rightarrow 2^{X}$ is called lower semicontinuous, if $S^{-1}(O):=\{x \in X: S x \cap O \neq \emptyset\}$ is open in $X$ whenever $O \subset Y$ is open.

We list some propositions and lemmas that will be used in the sequel.

Proposition 5 (see [12]). Let $\left\{\lambda_{n}\right\}$ and $\left\{b_{n}\right\}$ be sequences of nonnegative numbers and $\left\{\alpha_{n}\right\} \subset(0,1)$ a sequence satisfying the conditions that $\left\{\lambda_{n}\right\}$ is bounded, $\sum_{n=0}^{\infty} \alpha_{n}=\infty$, and $b_{n} \rightarrow$ 0 , as $n \rightarrow \infty$. Let the recursive inequality

$$
\lambda_{n+1}^{2} \leq \lambda_{n}^{2}-2 \alpha_{n} \psi\left(\lambda_{n+1}\right)+2 \alpha_{n} b_{n} \lambda_{n+1}, \quad \forall n \geq 0,
$$

be given where $\psi:[0, \infty) \rightarrow[0, \infty)$ is a strictly increasing function such that it is positive on $(0, \infty)$ and $\psi(0)=0$. Then $\lambda_{n} \rightarrow 0$, as $n \rightarrow \infty$.

Proposition 6 (see [13]). Let $X$ be a real smooth Banach space. Let $T$, and $F: X \rightarrow 2^{X}$ be two multivalued mappings, and let $N(\cdot, \cdot): X \times X \rightarrow X$ be a nonlinear mapping satisfying the following conditions:

(i) the mapping $x \mapsto N(x, y)$ is $\phi$-strongly accretive with respect to the mapping $T$;

(ii) the mapping $y \mapsto N(x, y)$ is accretive with respect to the mapping $F$.

Then the mapping $S: X \rightarrow 2^{X}$ defined by $S x=N(T x, F x)$ is $\phi$-strongly accretive.

Proposition 7 (see [14]). Let $X$ be a real Banach space and let $S: X \rightarrow 2^{X} \backslash\{\emptyset\}$ be a lower semicontinuous and $\phi$-strongly accretive mapping; then, for any $x \in X, S x$ is a one-point set; that is, $S$ is a single-valued mapping.

Lemma 8 can be found in [15]. Lemma 9 is an immediate consequence of the subdifferential inequality of the function $(1 / 2)\|\cdot\|^{2}$.

Lemma 8. Let $\left\{s_{n}\right\}$ be a sequence of nonnegative real numbers satisfying

$$
s_{n+1} \leq\left(1-\alpha_{n}\right) s_{n}+\alpha_{n} \beta_{n}+\gamma_{n}, \quad \forall n \geq 0,
$$

where $\left\{\alpha_{n}\right\},\left\{\beta_{n}\right\}$, and $\left\{\gamma_{n}\right\}$ satisfy the following conditions: (i) $\left\{\alpha_{n}\right\} \subset[0,1]$ and $\sum_{n=0}^{\infty} \alpha_{n}=\infty$;

(ii) $\limsup _{n \rightarrow \infty} \beta_{n} \leq 0$;

(iii) $\gamma_{n} \geq 0$, for all $n \geq 0$, and $\sum_{n=0}^{\infty} \gamma_{n}<\infty$.

Then $\lim \sup _{n \rightarrow \infty} s_{n}=0$.

Lemma 9. In a smooth Banach space $X$, there holds the inequality

$$
\|x+y\|^{2} \leq\|x\|^{2}+2\langle y, J(x+y)\rangle, \quad \forall x, y \in X .
$$

Lemma 10 (see [1]). Let $C$ be a nonempty closed convex subset of a smooth Banach space $X$. Let $\Pi_{C}$ be a sunny nonexpansive retraction from $X$ onto $C$ and let $A$ be an accretive operator of $C$ into $X$. Then, for all $\lambda>0$,

$$
\mathrm{VI}(C, A)=\operatorname{Fix}\left(\Pi_{C}(I-\lambda A)\right) .
$$

Let $D$ be a subset of $C$ and let $\Pi$ be a mapping of $C$ into $D$. Then $\Pi$ is said to be sunny if

$$
\Pi[\Pi(x)+t(x-\Pi(x))]=\Pi(x),
$$

whenever $\Pi(x)+t(x-\Pi(x)) \in C$ for $x \in C$ and $t \geq 0$. A mapping $\Pi$ of $C$ into itself is called a retraction if $\Pi^{2}=\Pi$. If a mapping $\Pi$ of $C$ into itself is a retraction, then $\Pi(z)=z$ for every $z \in R(\Pi)$ where $R(\Pi)$ is the range of $\Pi$. A subset $D$ of $C$ is called a sunny nonexpansive retract of $C$ if there exists a sunny nonexpansive retraction from $C$ onto $D$. The following lemma concerns the sunny nonexpansive retraction.

Lemma 11 (see [16]). Let C be a nonempty closed convex subset of a real smooth Banach space $X$. Let $D$ be a nonempty subset of $C$. Let $\Pi$ be a retraction of $C$ onto $D$. Then the following are equivalent:

(i) $\Pi$ is sunny and nonexpansive;

(ii) $\|\Pi(x)-\Pi(y)\|^{2} \leq\langle x-y, J(\Pi(x)-\Pi(y))\rangle$, for all $x, y \in C$;

(iii) $\langle x-\Pi(x), J(y-\Pi(x))\rangle \leq 0$, for all $x \in C, y \in D$.

It is well known that if $X=H$ a Hilbert space, then a sunny nonexpansive retraction $\Pi_{C}$ is coincident with the metric projection from $X$ onto $C$; that is, $\Pi_{C}=P_{C}$. If $C$ is a nonempty closed convex subset of a strictly convex and uniformly smooth Banach space $X$ and if $T: C \rightarrow C$ is a nonexpansive mapping with the fixed point set $\operatorname{Fix}(T) \neq \emptyset$, then the set $\operatorname{Fix}(T)$ is a sunny nonexpansive retract of $C$.

Lemma 12 (see [17]). Let $X$ be a uniformly convex Banach space and $\bar{B}_{r}(0):=\{x \in X:\|x\| \leq r\}, r>0$. Then there exists a continuous, strictly increasing, and convex function $\varphi:[0, \infty] \rightarrow[0, \infty], \varphi(0)=0$ such that

$$
\|\alpha x+\beta y+\gamma z\|^{2} \leq \alpha\|x\|^{2}+\beta\|y\|^{2}+\gamma\|z\|^{2}-\alpha \beta \varphi(\|x-y\|),
$$

for all $x, y, z \in \bar{B}_{r}(0)$ and all $\alpha, \beta, \gamma \in[0,1]$ with $\alpha+\beta+\gamma=1$. 
Lemma 13 (see [18]). Let $C$ be a nonempty closed convex subset of a Banach space $X$. Let $S_{0}, S_{1}, \ldots$ be a sequence of mappings of $C$ into itself. Suppose that $\sum_{n=1}^{\infty} \sup \left\{\left\|S_{n} x-S_{n-1} x\right\|\right.$ : $x \in C\}<\infty$. Then for each $y \in C,\left\{S_{n} y\right\}$ converges strongly to some point of $C$. Moreover, let $S$ be a mapping of $C$ into itself defined by $S y=\lim _{n \rightarrow \infty} S_{n} y$ for all $y \in C$. Then $\lim _{n \rightarrow \infty} \sup \left\{\left\|S x-S_{n} x\right\|: x \in C\right\}=0$.

Let $C$ be a nonempty closed convex subset of a Banach space $X$ and let $T: C \rightarrow C$ be a nonexpansive mapping with $\operatorname{Fix}(T) \neq \emptyset$. As previous, let $\Xi_{C}$ be the set of all contractions on $C$. For $t \in(0,1)$ and $f \in \Xi_{C}$, let $x_{t} \in C$ be the unique fixed point of the contraction $x \mapsto t f(x)+(1-t) T x$ on $C$; that is,

$$
x_{t}=t f\left(x_{t}\right)+(1-t) T x_{t}
$$

Lemma 14 (see [19]). Let $X$ be a uniformly smooth Banach space or a reflexive and strictly convex Banach space with a uniformly Gáteaux differentiable norm. Let $C$ be a nonempty closed convex subset of $X$, let $T: C \rightarrow C$ be a nonexpansive mapping with $\operatorname{Fix}(T) \neq \emptyset$, and let $f \in \Xi_{C}$. Then the net $\left\{x_{t}\right\}$ defined by $x_{t}=t f\left(x_{t}\right)+(1-t) T x_{t}$ converges strongly to $a$ point in $\operatorname{Fix}(T)$. If one defines a mapping $Q: \Xi_{C} \rightarrow \operatorname{Fix}(T)$ by $Q(f):=s-\lim _{t \rightarrow 0} x_{t}$, for all $f \in \Xi_{C}$, then $Q(f)$ solves the VIP as follows:

$$
\begin{array}{r}
\langle(I-f) Q(f), J(Q(f)-p)\rangle \leq 0, \\
\forall f \in \Xi_{C}, p \in \operatorname{Fix}(T) .
\end{array}
$$

Lemma 15 (see [20]). Let $C$ be a nonempty closed convex subset of a strictly convex Banach space $X$. Let $\left\{T_{n}\right\}_{n=0}^{\infty}$ be a sequence of nonexpansive mappings on $C$. Suppose $\bigcap_{n=0}^{\infty} \operatorname{Fix}\left(T_{n}\right)$ is nonempty. Let $\left\{\lambda_{n}\right\}$ be a sequence of positive numbers with $\sum_{n=0}^{\infty} \lambda_{n}=1$. Then a mapping $S$ on $C$ defined by $S x=\sum_{n=0}^{\infty} \lambda_{n} T_{n} x$ for $x \in C$ is defined well and nonexpansive, and $\operatorname{Fix}(S)=\bigcap_{n=0}^{\infty} \operatorname{Fix}\left(T_{n}\right)$ holds.

Lemma 16 (see [21]). Given a number $r>0$. A real Banach space $X$ is uniformly convex if and only if there exists a continuous strictly increasing function $\varphi:[0, \infty) \rightarrow[0, \infty)$, $\varphi(0)=0$, such that

$$
\begin{aligned}
& \|\lambda x+(1-\lambda) y\|^{2} \\
& \quad \leq \lambda\|x\|^{2}+(1-\lambda)\|y\|^{2}-\lambda(1-\lambda) \varphi(\|x-y\|),
\end{aligned}
$$

for all $\lambda \in[0,1]$ and $x, y \in X$ such that $\|x\| \leq r$ and $\|y\| \leq r$.

\section{Mann-Type Viscosity Algorithms in Uniformly Convex and 2-Uniformly Smooth Banach Spaces}

In this section, we introduce Mann-type viscosity iterative algorithms in uniformly convex and 2-uniformly smooth Banach spaces and show strong convergence theorems. We will use the following useful lemma.
Lemma 17. Let $C$ be a nonempty closed convex subset of a real 2-uniformly smooth Banach space X. Let $A: C \rightarrow X$ be an $\alpha$-inverse strongly accretive mapping. Then, one has

$$
\begin{aligned}
& \|(I-\lambda A) x-(I-\lambda A) y\|^{2} \\
& \quad \leq\|x-y\|^{2}+2 \lambda\left(\lambda \kappa^{2}-\alpha\right)\|A x-A y\|^{2}, \quad \forall x, y \in C,
\end{aligned}
$$

where $\lambda>0$. In particular, if $0<\lambda \leq \alpha / \kappa^{2}$, then $I-\lambda A$ is nonexpansive.

Theorem 18. Let $X$ be a uniformly convex and 2-uniformly smooth Banach space and let $C$ be a nonempty closed convex subset of $X$ such that $C \pm C \subset C$. Let $\Pi_{C}$ be a sunny nonexpansive retraction from $X$ onto $C$. Let $T, F: X \rightarrow$ $C B(X)$, and $A: C \rightarrow 2^{C}$ be three multivalued mappings, let $g: X \rightarrow C$ be a single-valued mapping, and let $N(\cdot, \cdot)$ : $X \times X \rightarrow C$ be a single-valued continuous mapping satisfying conditions (C1)-(C5) in Theorem 4. Consider that

(C6) $N(T x, F x)+A(g(x)): X \rightarrow 2^{C} \backslash\{\emptyset\}$ is $\zeta$-inverse strongly accretive with $\zeta \geq \kappa^{2}$.

Let $A_{i}: C \rightarrow X$ be an $\alpha_{i}$-inverse strongly accretive mapping for each $i=1, \ldots, N$. Define the mapping $G_{i}$ : $C \rightarrow C$ by $G_{i}=\Pi_{C}\left(I-\lambda_{i} A_{i}\right)$ for $i=1, \ldots, N$, where $\lambda_{i} \in\left(0, \alpha_{i} / \kappa^{2}\right)$ and $\kappa$ is the 2 -uniformly smooth constant of $X$. Let $B: C \rightarrow C$ be the $K$-mapping generated by $G_{1}, \ldots, G_{N}$ and $\rho_{1}, \ldots, \rho_{N}$, where $\rho_{i} \in(0,1)$, for all $i=1, \ldots, N-1$ and $\rho_{N} \in(0,1]$. Let $f: C \rightarrow C$ be a contraction with coefficient $\rho \in(0,1)$. Let $\left\{S_{i}\right\}_{i=0}^{\infty}$ be a countable family of nonexpansive mappings of $C$ into itself such that $\Delta:=\bigcap_{i=0}^{\infty} \operatorname{Fix}\left(S_{i}\right) \cap \Gamma \cap$ $\left(\cap_{i=1}^{N} \operatorname{VI}\left(C, A_{i}\right)\right) \neq \emptyset$. Suppose that $\left\{\alpha_{n}\right\},\left\{\beta_{n}\right\},\left\{\gamma_{n}\right\},\left\{\delta_{n}\right\},\left\{\sigma_{n}\right\}$, and $\left\{\epsilon_{n}\right\}$ are the sequences in $[0,1], \alpha_{n}+\beta_{n}+\gamma_{n}+\delta_{n}=1$ and satisfy the following conditions:

(i) $\sum_{n=1}^{\infty}\left(\left|\alpha_{n}-\alpha_{n-1}\right|+\left|\beta_{n}-\beta_{n-1}\right|+\left|\gamma_{n}-\gamma_{n-1}\right|+\left|\delta_{n}-\delta_{n-1}\right|+\right.$ $\left.\left|\sigma_{n}-\sigma_{n-1}\right|+\left|\epsilon_{n}-\epsilon_{n-1}\right|\right)<\infty$;

(ii) $\lim _{n \rightarrow \infty} \alpha_{n}=0$ and $\sum_{n=0}^{\infty} \alpha_{n}=\infty$;

(iii) $\left\{\gamma_{n}\right\},\left\{\delta_{n}\right\} \subset[c, d]$ for some $c, d \in(0,1)$;

(iv) $0<\liminf _{n \rightarrow \infty} \beta_{n} \leq \limsup _{n \rightarrow \infty} \beta_{n}<1$;

(v) $0<\liminf _{n \rightarrow \infty} \sigma_{n} \leq \limsup _{n \rightarrow \infty} \sigma_{n}<1$;

(vi) $0<\liminf _{n \rightarrow \infty} \epsilon_{n} \leq \limsup _{n \rightarrow \infty} \epsilon_{n}<1$.

For arbitrary $x_{0} \in C$, define the sequence $\left\{x_{n}\right\}$ iteratively by

$$
\begin{aligned}
y_{n}= & \alpha_{n} f\left(x_{n}\right)+\beta_{n} x_{n}+\gamma_{n} B x_{n}+\delta_{n} S_{n} x_{n}, \\
x_{n+1}= & \epsilon_{n}\left[x_{n}-\sigma_{n}\left(N\left(w_{n}, k_{n}\right)+u_{n}\right)\right] \\
& +\left(1-\epsilon_{n}\right) y_{n}, \quad u_{n} \in A\left(g\left(x_{n}\right)\right), \quad \forall n \geq 0,
\end{aligned}
$$

where $\left\{u_{n}\right\}$ is defined by

$$
\begin{array}{r}
\left\|u_{n}-u_{n+1}\right\| \leq(1+\varepsilon) H\left(A\left(g\left(x_{n+1}\right)\right), A\left(g\left(x_{n}\right)\right)\right), \\
\forall n \geq 0,
\end{array}
$$

for any $w_{n} \in T x_{n}, k_{n} \in F x_{n}$, and some $\varepsilon>0$. Assume that $\sum_{n=0}^{\infty} \sup _{x \in D}\left\|S_{n+1} x-S_{n} x\right\|<\infty$ for any bounded subset 
$D$ of $C$ and let $S$ be a mapping of $C$ into itself defined by $S x=\lim _{n \rightarrow \infty} S_{n} x$ for all $x \in C$ and suppose that $\operatorname{Fix}(S)=$ $\bigcap_{i=0}^{\infty} \operatorname{Fix}\left(S_{i}\right)$. Then $\left\{x_{n}\right\}$ converges strongly to $q \in \Delta$, which solves the following VIP:

$$
\langle q-f(q), J(q-p)\rangle \leq 0, \quad \forall p \in \Delta,
$$

and, for any $w \in T q$ and $k \in F q,(q, w, k)$ is a solution of the MVVI (16).

Proof. First of all, by Lemma 17 we know that $I-\lambda_{i} A_{i}$ is a nonexpansive mapping, where $\lambda_{i} \in\left(0, \alpha_{i} / \kappa^{2}\right)$ for each $i=1, \ldots, N$. Hence, from the nonexpansivity of $\Pi_{C}$, it follows that $G_{i}$ is a nonexpansive mapping for each $i=$ $1, \ldots, N$. Since $B: C \rightarrow C$ is the $K$-mapping generated by $G_{1}, \ldots, G_{N}$ and $\rho_{1}, \ldots, \rho_{N}$, by Lemma 3 , we deduce that $\operatorname{Fix}(B)=\cap_{i=1}^{N} \operatorname{Fix}\left(G_{i}\right)$. Utilizing Lemma 10, and the definition of $G_{i}$, we get $\operatorname{Fix}\left(G_{i}\right)=\operatorname{VI}\left(C, A_{i}\right)$ for each $i=1, \ldots, N$. Thus, we have

$$
\operatorname{Fix}(B)=\bigcap_{i=1}^{N} \operatorname{Fix}\left(G_{i}\right)=\bigcap_{i=1}^{N} \operatorname{VI}\left(C, A_{i}\right)
$$

Now, let us show that for any $v \in C, \lambda>0$, there exists a point $\tilde{x} \in C$ such that $(\tilde{x}, w, k)$ is a solution of the MVVI (15), for any $w \in T \tilde{x}$ and $k \in F \tilde{x}$. Indeed, following the argument idea in the proof of Chidume et al. [5, Theorem 3.1], we put $V x:=N(T x, F x)$ for all $x \in X$. Then by Proposition $6, V$ is $\phi$ strongly accretive. Since $T$ and $F$ are $H$-uniformly continuous and $N(\cdot, \cdot)$ is continuous, $V x$ is continuous and hence lower semicontinuous. Thus, by Proposition 7, Vx is single-valued. Moreover, since $V$ is $\phi$-strongly accretive and by assumption $A \circ g: X \rightarrow 2^{C}$ is $m$-accretive, we have that $V+\lambda A \circ g$ is an $m$-accretive and $\phi$-strongly accretive mapping, and hence by Cioranescu [22, page 184], for any $x \in X$, we have that $(V+$ $\lambda A \circ g)(x)$ is closed and bounded. Therefore, by Morales [23], $V+\lambda A \circ g$ is surjective. Hence, for any $v \in X$ and $\lambda>0$, there exists $\tilde{x} \in D(A)=C$ such that $v \in V \tilde{x}+\lambda A(g(\tilde{x}))=N(w, k)+$ $\lambda A(g(\tilde{x}))$, where $w \in T \tilde{x}$ and $k \in F \tilde{x}$. In addition, in terms of Proposition 7, we know that $V+\lambda A \circ g$ is a single-valued mapping. Assume that $N(T x, F x)+\lambda A(g(x)): X \rightarrow C$ is $\zeta$ inverse strongly accretive with $\zeta \geq \kappa^{2}$. Then by Lemma 17, we conclude that the mapping $x \mapsto x-(N(T x, F x)+\lambda A(g(x)))$ is nonexpansive.

Without loss of generality, we may assume that $v=0$ and $\lambda=1$. Let $p \in \Delta$ and let $r(\geq\|f(p)-p\| /(1-\rho))$ be sufficiently large such that $x_{0} \in \bar{B}_{r}(p)=: B$. Then $p \in D(A)=C$ such that $0 \in N(w, k)+A \circ g(p)$ for any $w \in T p$ and $k \in F p$. Let $M:=\sup \{\|u\|: u \in N(w, k)+A(g(x)), x \in B, w \in T x$, $k \in F x\}$. Then as $A \circ g, T$, and $F$ are $H$-uniformly continuous on $X$, for $\varepsilon_{1}:=\phi(r) / 8(1+\varepsilon), \varepsilon_{2}:=\phi(r) / 8 \mu(1+\varepsilon)$, and $\varepsilon_{3}:=$ $\phi(r) / 8 \xi(1+\varepsilon)$, there exist $\delta_{1}, \delta_{2}, \delta_{3}>0$ such that for any $x, y \in X,\|x-y\|<\delta_{1},\|x-y\|<\delta_{2}$ and $\|x-y\|<\delta_{3}$ imply $H(A \circ g(x), A \circ g(y))<\varepsilon_{1}, H(T x, T y)<\varepsilon_{2}$ and $H(F x, F y)<$ $\varepsilon_{3}$, respectively.

Let us show that $x_{n} \in B$ for all $n \geq 0$. We show this by induction. First, $x_{0} \in B$ by construction. Assume that $x_{n} \in B$.
We show that $x_{n+1} \in B$. If possible we assume that $x_{n+1} \notin B$, then $\left\|x_{n+1}-p\right\|>r$. Further from (35) it follows that

$$
\begin{aligned}
\| y_{n}- & p \| \\
= & \| \alpha_{n}\left(f\left(x_{n}\right)-p\right)+\beta_{n}\left(x_{n}-p\right) \\
& +\gamma_{n}\left(B x_{n}-p\right)+\delta_{n}\left(S_{n} x_{n}-p\right) \| \\
\leq & \alpha_{n}\left\|f\left(x_{n}\right)-p\right\|+\beta_{n}\left\|x_{n}-p\right\| \\
& +\gamma_{n}\left\|B x_{n}-p\right\|+\delta_{n}\left\|S_{n} x_{n}-p\right\| \\
\leq & \alpha_{n}\left(\left\|f\left(x_{n}\right)-f(p)\right\|+\|f(p)-p\|\right) \\
& +\beta_{n}\left\|x_{n}-p\right\|+\gamma_{n}\left\|B x_{n}-p\right\|+\delta_{n}\left\|S_{n} x_{n}-p\right\| \\
\leq & \alpha_{n}\left(\rho\left\|x_{n}-p\right\|+\|f(p)-p\|\right) \\
& +\beta_{n}\left\|x_{n}-p\right\|+\gamma_{n}\left\|x_{n}-p\right\|+\delta_{n}\left\|x_{n}-p\right\| \\
= & \left(1-\alpha_{n}(1-\rho)\right)\left\|x_{n}-p\right\|+\alpha_{n}\|f(p)-p\| \\
= & \left(1-\alpha_{n}(1-\rho)\right)\left\|x_{n}-p\right\| \\
& +\alpha_{n}(1-\rho) \frac{\|f(p)-p\|}{1-\rho} \\
\leq & \max \left\{\left\|x_{n}-p\right\|, \frac{\|f(p)-p\|}{1-\rho}\right\},
\end{aligned}
$$

and hence

$$
\begin{aligned}
\| x_{n+1}- & p \|^{2} \\
= & \left\langle\epsilon_{n}\left[x_{n}-p-\sigma_{n}\left(N\left(w_{n}, k_{n}\right)+u_{n}\right)\right]\right. \\
& \left.+\left(1-\epsilon_{n}\right)\left(y_{n}-p\right), J\left(x_{n+1}-p\right)\right\rangle \\
= & \left\langle\epsilon_{n}\left(x_{n}-p\right)+\left(1-\epsilon_{n}\right)\left(y_{n}-p\right), J\left(x_{n+1}-p\right)\right\rangle \\
& -\epsilon_{n} \sigma_{n}\left\langle N\left(w_{n}, k_{n}\right)+u_{n}, J\left(x_{n+1}-p\right)\right\rangle \\
\leq & \left\|\alpha_{n}\left(x_{n}-p\right)+\left(1-\alpha_{n}\right)\left(y_{n}-p\right)\right\|\left\|x_{n+1}-p\right\| \\
& -\epsilon_{n} \sigma_{n}\left\langle N\left(w_{n}, k_{n}\right)+u_{n}, J\left(x_{n+1}-p\right)\right\rangle \\
\leq & \left(\epsilon_{n}\left\|x_{n}-p\right\|+\left(1-\epsilon_{n}\right)\left\|y_{n}-p\right\|\right)\left\|x_{n+1}-p\right\| \\
& -\alpha_{n} \sigma_{n}\left\langle N\left(w_{n}, k_{n}\right)+u_{n}, J\left(x_{n+1}-p\right)\right\rangle \\
\leq & \left(\epsilon_{n}\left\|x_{n}-p\right\|+\left(1-\epsilon_{n}\right)\right. \\
& \quad-\alpha_{n} \sigma_{n}\left\langle N\left(w_{n}, k_{n}\right)+u_{n}, J\left(x_{n+1}-p\right)\right\rangle \\
& \left.\max \left\{\left\|x_{n}-p\right\|, \frac{\|f(p)-p\|\}}{1-\rho}\right\}\right)\left\|x_{n+1}-p\right\| \\
- & \alpha_{n} \sigma_{n}\left\langle N\left(w_{n}, k_{n}\right)+u_{n}, J\left(x_{n+1}-p\right)\right\rangle \\
& \max \left\{\left\|x_{n}-p\right\|, \frac{\|f(p)-p\|}{1-\rho}\right\}\left\|x_{n+1}-p\right\|
\end{aligned}
$$




$$
\begin{aligned}
\leq & \frac{1}{2}\left(\max \left\{\left\|x_{n}-p\right\|^{2},\left\|\frac{f(p)-p}{1-\rho}\right\|^{2}\right\}+\left\|x_{n+1}-p\right\|^{2}\right) \\
& -\alpha_{n} \sigma_{n}\left\langle N\left(w_{n}, k_{n}\right)+u_{n}, J\left(x_{n+1}-p\right)\right\rangle,
\end{aligned}
$$

which immediately yields

$$
\begin{aligned}
& \left\|x_{n+1}-p\right\|^{2} \\
& \leq \max \left\{\left\|x_{n}-p\right\|^{2},\left\|\frac{f(p)-p}{1-\rho}\right\|^{2}\right\} \\
& \quad-2 \alpha_{n} \sigma_{n}\left\langle N\left(w_{n}, k_{n}\right)+u_{n}, J\left(x_{n+1}-p\right)\right\rangle \\
& =\max \left\{\left\|x_{n}-p\right\|^{2},\left\|\frac{f(p)-p}{1-\rho}\right\|^{2}\right\} \\
& \quad-2 \alpha_{n} \sigma_{n}\left\langle N\left(w_{n+1}, k_{n+1}\right)+u_{n+1}, J\left(x_{n+1}-p\right)\right\rangle \\
& \quad-2 \alpha_{n} \sigma_{n}\left\langle N\left(w_{n}, k_{n}\right)+u_{n}\right. \\
& \left.\quad-\left(N\left(w_{n+1}, k_{n+1}\right)+u_{n+1}\right), J\left(x_{n+1}-p\right)\right\rangle .
\end{aligned}
$$

Since $N(\cdot, \cdot)$ is $\phi$-strongly accretive with respect to $T$ and $A(g(\cdot))$ is accretive, we deduce from (41) that

$$
\begin{aligned}
& \left\|x_{n+1}-p\right\|^{2} \\
& \leq \max \left\{\left\|x_{n}-p\right\|^{2},\left\|\frac{f(p)-p}{1-\rho}\right\|^{2}\right\} \\
& -2 \alpha_{n} \sigma_{n} \phi\left(\left\|x_{n+1}-p\right\|\right)\left\|x_{n+1}-p\right\| \\
& +2 \alpha_{n} \sigma_{n}\left[\left\|N\left(w_{n+1}, k_{n+1}\right)-N\left(w_{n}, k_{n}\right)\right\|\right. \\
& \left.\quad+\left\|u_{n+1}-u_{n}\right\|\right]\left\|x_{n+1}-p\right\| \\
& \leq \max \left\{\left\|x_{n}-p\right\|^{2},\left\|\frac{f(p)-p \|^{2}}{1-\rho}\right\|^{2}\right\} \\
& +2 \alpha_{n} \sigma_{n} \phi\left(\left\|x_{n+1}-p\right\|\right)\left\|x_{n+1}-p\right\| \\
& +2 \alpha_{n} \sigma_{n}\left[\left\|N\left(w_{n+1}, k_{n+1}\right)-N\left(w_{n+1}, k_{n}\right)\right\|\right. \\
& +\left\|N\left(w_{n+1}, k_{n}\right)-N\left(w_{n}, k_{n}\right)\right\| \\
& \left.+\left\|u_{n+1}-u_{n}\right\|\right]\left\|x_{n+1}-p\right\| .
\end{aligned}
$$

Again from (35), we have that

$$
\begin{aligned}
& \left\|x_{n+1}-p\right\| \\
& \quad \leq \epsilon_{n}\left\|x_{n}-p-\sigma_{n}\left(N\left(w_{n}, k_{n}\right)+u_{n}\right)\right\| \\
& \quad+\left(1-\epsilon_{n}\right)\left\|y_{n}-p\right\|
\end{aligned}
$$

$$
\begin{aligned}
& \leq \epsilon_{n}\left[\left\|x_{n}-p\right\|+\sigma_{n}\left\|N\left(w_{n}, k_{n}\right)+u_{n}\right\|\right] \\
& \quad+\left(1-\epsilon_{n}\right) \max \left\{\left\|x_{n}-p\right\|, \frac{\|f(p)-p\|}{1-\rho}\right\} \\
& \leq \epsilon_{n}\left[r+\sigma_{n} M\right]+\left(1-\epsilon_{n}\right) r \\
& \leq 2 r .
\end{aligned}
$$

Also, from Proposition 7, $V x=N(T x, F x)$ is a single-valued mapping; that is, for any $k, k^{\prime} \in F x$ and $w, w^{\prime} \in T x$, we have $N(w, k)=N\left(w, k^{\prime}\right)$ and $N(w, k)=N\left(w^{\prime}, k\right)$. On the other hand, it follows from Nadler [24] that, for $k_{n+1} \in F x_{n+1}$ and $w_{n+1} \in T x_{n+1}$, there exist $k_{n}^{\prime} \in F x_{n}$ and $w_{n}^{\prime} \in T x_{n}$ such that

$$
\begin{aligned}
& \left\|k_{n+1}-k_{n}^{\prime}\right\| \leq(1+\varepsilon) H\left(F x_{n+1}, F x_{n}\right), \\
& \left\|w_{n+1}-w_{n}^{\prime}\right\| \leq(1+\varepsilon) H\left(T x_{n+1}, T x_{n}\right),
\end{aligned}
$$

respectively. Therefore, from (42) and (36), we have

$$
\begin{aligned}
& \left\|x_{n+1}-p\right\|^{2} \\
& \leq \max \left\{\left\|x_{n}-p\right\|^{2},\left\|\frac{f(p)-p}{1-\rho}\right\|^{2}\right\}-2 \alpha_{n} \sigma_{n} \phi(r) r \\
& +2 \alpha_{n} \sigma_{n}\left[\left\|N\left(w_{n+1}, k_{n+1}\right)-N\left(w_{n+1}, k_{n}^{\prime}\right)\right\|\right. \\
& +\left\|N\left(w_{n+1}, k_{n}\right)-N\left(w_{n}^{\prime}, k_{n}\right)\right\| \\
& \left.+\left\|u_{n+1}-u_{n}\right\|\right] 2 r \\
& \leq \max \left\{\left\|x_{n}-p\right\|^{2},\left\|\frac{f(p)-p}{1-\rho}\right\|^{2}\right\}-2 \alpha_{n} \sigma_{n} \phi(r) r \\
& +2 \alpha_{n} \sigma_{n}\left[\xi(1+\varepsilon) H\left(F x_{n+1}, F x_{n}\right)\right. \\
& +\mu(1+\varepsilon) H\left(T x_{n+1}, T x_{n}\right) \\
& \left.+(1+\varepsilon) H\left(A\left(g\left(x_{n+1}\right)\right), A\left(g\left(x_{n}\right)\right)\right)\right] 2 r \\
& \leq \max \left\{\left\|x_{n}-p\right\|^{2},\left\|\frac{f(p)-p}{1-\rho}\right\|^{2}\right\}-2 \alpha_{n} \sigma_{n} \phi(r) r \\
& +2 \alpha_{n} \sigma_{n}\left[\frac{\phi(r)}{8}+\frac{\phi(r)}{8}+\frac{\phi(r)}{8}\right] 2 r \\
& =\max \left\{\left\|x_{n}-p\right\|^{2},\left\|\frac{f(p)-p}{1-\rho}\right\|^{2}\right\} \\
& -2 \alpha_{n} \sigma_{n} \phi(r) r+\alpha_{n} \sigma_{n} \frac{3}{2} \phi(r) r \\
& \leq \max \left\{\left\|x_{n}-p\right\|^{2},\left\|\frac{f(p)-p}{1-\rho}\right\|^{2}\right\} \text {. }
\end{aligned}
$$


So, we get $\left\|x_{n+1}-p\right\| \leq r$, a contradiction. Therefore, $\left\{x_{n}\right\}$ is bounded.

Let us show that $\lim _{n \rightarrow \infty}\left\|x_{n}-x_{n+1}\right\|=0$ and $\lim _{n \rightarrow \infty}\left\|x_{n}-y_{n}\right\|=0$.

Indeed, we define $G: C \rightarrow C$ by $G x:=x-(N(T x, F x)+$ $A(g(x)))$ for all $x \in C$. Then, $G$ is a nonexpansive mapping and the iterative scheme (35) can be rewritten as follows:

$$
\begin{gathered}
y_{n}=\alpha_{n} f\left(x_{n}\right)+\beta_{n} x_{n}+\gamma_{n} B x_{n}+\delta_{n} S_{n} x_{n}, \\
x_{n+1}=\epsilon_{n}\left[\left(1-\sigma_{n}\right) x_{n}+\sigma_{n} G x_{n}\right] \\
+\left(1-\epsilon_{n}\right) y_{n}, \quad \forall n \geq 0 .
\end{gathered}
$$

Taking into account condition (iv), we may assume that $\left\{\beta_{n}\right\} \subset[a, b]$ for some $a, b \in(0,1)$. From (47), we can rewrite $y_{n}$ by

$$
y_{n}=\beta_{n} x_{n}+\left(1-\beta_{n}\right) z_{n}
$$

where $z_{n}=\left(\alpha_{n} f\left(x_{n}\right)+\gamma_{n} B x_{n}+\delta_{n} S_{n} x_{n}\right) /\left(1-\beta_{n}\right)$. Now, we have

$$
\begin{aligned}
& \left\|z_{n+1}-z_{n}\right\| \\
& =\| \frac{\alpha_{n+1} f\left(x_{n+1}\right)+\gamma_{n+1} B x_{n+1}+\delta_{n+1} S_{n+1} x_{n+1}}{1-\beta_{n+1}} \\
& -\frac{\alpha_{n} f\left(x_{n}\right)+\gamma_{n} B x_{n}+\delta_{n} S_{n} x_{n}}{1-\beta_{n}} \| \\
& =\left\|\frac{y_{n+1}-\beta_{n+1} x_{n+1}}{1-\beta_{n+1}}-\frac{y_{n}-\beta_{n} x_{n}}{1-\beta_{n}}\right\| \\
& =\| \frac{y_{n+1}-\beta_{n+1} x_{n+1}}{1-\beta_{n+1}}-\frac{y_{n}-\beta_{n} x_{n}}{1-\beta_{n+1}} \\
& +\frac{y_{n}-\beta_{n} x_{n}}{1-\beta_{n+1}}-\frac{y_{n}-\beta_{n} x_{n}}{1-\beta_{n}} \| \\
& \leq\left\|\frac{y_{n+1}-\beta_{n+1} x_{n+1}}{1-\beta_{n+1}}-\frac{y_{n}-\beta_{n} x_{n}}{1-\beta_{n+1}}\right\| \\
& +\left\|\frac{y_{n}-\beta_{n} x_{n}}{1-\beta_{n+1}}-\frac{y_{n}-\beta_{n} x_{n}}{1-\beta_{n}}\right\| \\
& =\frac{1}{1-\beta_{n+1}}\left\|y_{n+1}-\beta_{n+1} x_{n+1}-\left(y_{n}-\beta_{n} x_{n}\right)\right\| \\
& +\left|\frac{1}{1-\beta_{n+1}}-\frac{1}{1-\beta_{n}}\right|\left\|y_{n}-\beta_{n} x_{n}\right\| \\
& =\frac{1}{1-\beta_{n+1}}\left\|y_{n+1}-\beta_{n+1} x_{n+1}-\left(y_{n}-\beta_{n} x_{n}\right)\right\| \\
& +\frac{\left|\beta_{n+1}-\beta_{n}\right|}{\left(1-\beta_{n}\right)\left(1-\beta_{n+1}\right)}\left\|y_{n}-\beta_{n} x_{n}\right\|
\end{aligned}
$$

$$
=\frac{1}{1-\beta_{n+1}} \| \alpha_{n+1} f\left(x_{n+1}\right)+\gamma_{n+1} B x_{n+1}+\delta_{n+1} S_{n+1} x_{n+1}
$$$$
-\left(\alpha_{n} f\left(x_{n}\right)+\gamma_{n} B x_{n}+\delta_{n} S_{n} x_{n}\right) \|
$$$$
+\frac{\left|\beta_{n+1}-\beta_{n}\right|}{\left(1-\beta_{n}\right)\left(1-\beta_{n+1}\right)}\left\|y_{n}-\beta_{n} x_{n}\right\|
$$$$
\leq \frac{1}{1-\beta_{n+1}}\left(\alpha_{n+1}\left\|f\left(x_{n+1}\right)-f\left(x_{n}\right)\right\|\right.
$$$$
+\gamma_{n+1}\left\|B x_{n+1}-B x_{n}\right\|
$$$$
+\delta_{n+1}\left\|S_{n+1} x_{n+1}-S_{n} x_{n}\right\|
$$$$
+\left|\alpha_{n+1}-\alpha_{n}\right|\left\|f\left(x_{n}\right)\right\|
$$$$
\left.+\left|\gamma_{n+1}-\gamma_{n}\right|\left\|B x_{n}\right\|+\left|\delta_{n+1}-\delta_{n}\right|\left\|S_{n} x_{n}\right\|\right)
$$$$
+\frac{\left|\beta_{n+1}-\beta_{n}\right|}{\left(1-\beta_{n}\right)\left(1-\beta_{n+1}\right)}\left\|y_{n}-\beta_{n} x_{n}\right\|
$$$$
\leq \frac{1}{1-\beta_{n+1}}\left[\alpha_{n+1}\left\|f\left(x_{n+1}\right)-f\left(x_{n}\right)\right\|+\gamma_{n+1}\left\|x_{n+1}-x_{n}\right\|\right.
$$$$
+\delta_{n+1}\left(\left\|S_{n+1} x_{n+1}-S_{n+1} x_{n}\right\|\right.
$$$$
\left.+\left\|S_{n+1} x_{n}-S_{n} x_{n}\right\|\right)
$$$$
+\left|\alpha_{n+1}-\alpha_{n}\right|\left\|f\left(x_{n}\right)\right\|+\left|\gamma_{n+1}-\gamma_{n}\right|
$$$$
\left.\times\left\|B x_{n}\right\|+\left|\delta_{n+1}-\delta_{n}\right|\left\|S_{n} x_{n}\right\|\right]
$$$$
+\frac{\left|\beta_{n+1}-\beta_{n}\right|}{\left(1-\beta_{n}\right)\left(1-\beta_{n+1}\right)}\left\|y_{n}-\beta_{n} x_{n}\right\|
$$$$
\leq \frac{1}{1-\beta_{n+1}}\left[\alpha_{n+1} \rho\left\|x_{n+1}-x_{n}\right\|+\gamma_{n+1}\left\|x_{n+1}-x_{n}\right\|\right.
$$$$
+\delta_{n+1}\left(\left\|x_{n+1}-x_{n}\right\|+\left\|S_{n+1} x_{n}-S_{n} x_{n}\right\|\right)
$$$$
+\left|\alpha_{n+1}-\alpha_{n}\right|\left\|f\left(x_{n}\right)\right\|
$$$$
\left.+\left|\gamma_{n+1}-\gamma_{n}\right|\left\|B x_{n}\right\|+\left|\delta_{n+1}-\delta_{n}\right|\left\|S_{n} x_{n}\right\|\right]
$$$$
+\frac{\left|\beta_{n+1}-\beta_{n}\right|}{\left(1-\beta_{n}\right)\left(1-\beta_{n+1}\right)}\left\|\alpha_{n} f\left(x_{n}\right)+\gamma_{n} B x_{n}+\delta_{n} S_{n} x_{n}\right\|
$$$$
=\frac{1-\beta_{n+1}-\alpha_{n+1}(1-\rho)}{1-\beta_{n+1}}\left\|x_{n+1}-x_{n}\right\|
$$$$
+\frac{\delta_{n+1}}{1-\beta_{n+1}}\left\|S_{n+1} x_{n}-S_{n} x_{n}\right\|
$$$$
+\frac{1}{1-\beta_{n+1}}\left[\left|\alpha_{n+1}-\alpha_{n}\right|\left\|f\left(x_{n}\right)\right\|+\left|\gamma_{n+1}-\gamma_{n}\right|\right.
$$$$
\left.\times\left\|B x_{n}\right\|+\left|\delta_{n+1}-\delta_{n}\right|\left\|S_{n} x_{n}\right\|\right]
$$$$
+\frac{\left|\beta_{n+1}-\beta_{n}\right|}{\left(1-\beta_{n}\right)\left(1-\beta_{n+1}\right)}\left\|\alpha_{n} f\left(x_{n}\right)+\gamma_{n} B x_{n}+\delta_{n} S_{n} x_{n}\right\|
$$ 


$$
\begin{gathered}
\leq\left(1-\frac{\alpha_{n+1}(1-\rho)}{1-\beta_{n+1}}\right)\left\|x_{n+1}-x_{n}\right\|+\left\|S_{n+1} x_{n}-S_{n} x_{n}\right\| \\
+M_{0}\left[\left|\alpha_{n+1}-\alpha_{n}\right|+\left|\beta_{n+1}-\beta_{n}\right|\right. \\
\left.\quad+\left|\gamma_{n+1}-\gamma_{n}\right|+\left|\delta_{n+1}-\delta_{n}\right|\right],
\end{gathered}
$$

where $1 /(1-b)^{2} \sup _{n \geq 0}\left\{\left\|f\left(x_{n}\right)\right\|+\left\|B x_{n}\right\|+\left\|S_{n} x_{n}\right\|\right\} \leq M_{0}$ for some $M_{0}>0$. By simple calculation, we have

$$
\begin{aligned}
y_{n}-y_{n-1}= & \beta_{n}\left(x_{n}-x_{n-1}\right)+\left(\beta_{n}-\beta_{n-1}\right) \\
& \times\left(x_{n-1}-z_{n-1}\right)+\left(1-\beta_{n}\right)\left(z_{n}-z_{n-1}\right) .
\end{aligned}
$$

So, from (49), we get

$$
\begin{aligned}
& \left\|y_{n}-y_{n-1}\right\| \\
& \leq \beta_{n}\left\|x_{n}-x_{n-1}\right\|+\left|\beta_{n}-\beta_{n-1}\right|\left\|x_{n-1}-z_{n-1}\right\| \\
& \quad+\left(1-\beta_{n}\right)\left\|z_{n}-z_{n-1}\right\| \\
& \leq \beta_{n}\left\|x_{n}-x_{n-1}\right\|+\left|\beta_{n}-\beta_{n-1}\right|\left\|x_{n-1}-z_{n-1}\right\| \\
& \quad+\left(1-\beta_{n}\right)\left\{\left(1-\frac{\alpha_{n}(1-\rho)}{1-\beta_{n}}\right)\left\|x_{n}-x_{n-1}\right\|\right. \\
& \quad+\left\|S_{n} x_{n-1}-S_{n-1} x_{n-1}\right\| \\
& \quad+M_{0}\left[\left|\alpha_{n}-\alpha_{n-1}\right|+\left|\beta_{n}-\beta_{n-1}\right|\right. \\
& \left.\left.\quad+\left|\gamma_{n}-\gamma_{n-1}\right|+\left|\delta_{n}-\delta_{n-1}\right|\right]\right\} \\
& \quad+\left(1-\alpha_{n}(1-\rho)\right)\left\|x_{n}-x_{n-1}\right\| \\
& +\left|\beta_{n}-\beta_{n-1}\right|\left\|x_{n-1}-z_{n-1}\right\| \\
& +|| S_{n} x_{n-1}-S_{n-1} x_{n-1} \| \\
& +M_{0}\left[\left|\alpha_{n}-\alpha_{n-1}\right|+\left|\beta_{n}-\beta_{n-1}\right|\right. \\
& \left.+\left|\gamma_{n}-\gamma_{n-1}\right|+\left|\delta_{n}-\delta_{n-1}\right|\right] .
\end{aligned}
$$

Also, for convenience, we write

$$
\begin{aligned}
& x_{n+1}=\epsilon_{n} \widehat{z}_{n}+\left(1-\epsilon_{n}\right) y_{n}, \\
& \widehat{z}_{n}=\sigma_{n} G x_{n}+\left(1-\sigma_{n}\right) x_{n} .
\end{aligned}
$$

By simple calculation, we get

$$
\begin{aligned}
x_{n+1}-x_{n}= & \epsilon_{n}\left(\widehat{z}_{n}-\widehat{z}_{n-1}\right)+\left(\epsilon_{n}-\epsilon_{n-1}\right) \\
& \times\left(\widehat{z}_{n-1}-y_{n-1}\right)+\left(1-\epsilon_{n}\right)\left(y_{n}-y_{n-1}\right), \\
\widehat{z}_{n}-\widehat{z}_{n-1}= & \sigma_{n}\left(G x_{n}-G x_{n-1}\right)+\left(\sigma_{n}-\sigma_{n-1}\right) \\
& \times\left(G x_{n-1}-x_{n-1}\right)+\left(1-\sigma_{n}\right)\left(x_{n}-x_{n-1}\right) .
\end{aligned}
$$

From (51) and (53), we deduce that

$$
\begin{aligned}
&\left\|\bar{z}_{n}-\widehat{z}_{n-1}\right\| \\
& \leq \sigma_{n}\left\|G x_{n}-G x_{n-1}\right\|+\left|\sigma_{n}-\sigma_{n-1}\right| \\
& \quad \times\left\|G x_{n-1}-x_{n-1}\right\|+\left(1-\sigma_{n}\right)\left\|x_{n}-x_{n-1}\right\| \\
& \leq \sigma_{n}\left\|x_{n}-x_{n-1}\right\|+\left|\sigma_{n}-\sigma_{n-1}\right| \\
& \times\left\|G x_{n-1}-x_{n-1}\right\|+\left(1-\sigma_{n}\right)\left\|x_{n}-x_{n-1}\right\| \\
& \leq\left\|x_{n}-x_{n-1}\right\|+\left|\sigma_{n}-\sigma_{n-1}\right|\left\|G x_{n-1}-x_{n-1}\right\|,
\end{aligned}
$$

and hence

$$
\begin{aligned}
& \left\|x_{n+1}-x_{n}\right\| \\
& \leq \epsilon_{n}\left\|\widehat{z}_{n}-\widehat{z}_{n-1}\right\|+\left|\epsilon_{n}-\epsilon_{n-1}\right| \\
& \times\left\|\widehat{z}_{n-1}-y_{n-1}\right\|+\left(1-\epsilon_{n}\right)\left\|y_{n}-y_{n-1}\right\| \\
& \leq \epsilon_{n}\left[\left\|x_{n}-x_{n-1}\right\|+\left|\sigma_{n}-\sigma_{n-1}\right|\left\|G x_{n-1}-x_{n-1}\right\|\right] \\
& +\left|\epsilon_{n}-\epsilon_{n-1}\right||| \widehat{z}_{n-1}-y_{n-1} \| \\
& +\left(1-\epsilon_{n}\right)\left\{\left(1-\alpha_{n}(1-\rho)\right)\left\|x_{n}-x_{n-1}\right\|\right. \\
& +\left|\beta_{n}-\beta_{n-1}\right|\left\|x_{n-1}-z_{n-1}\right\| \\
& +\left\|S_{n} x_{n-1}-S_{n-1} x_{n-1}\right\| \\
& +M_{0}\left[\left|\alpha_{n}-\alpha_{n-1}\right|+\left|\beta_{n}-\beta_{n-1}\right|\right. \\
& \left.\left.+\left|\gamma_{n}-\gamma_{n-1}\right|+\left|\delta_{n}-\delta_{n-1}\right|\right]\right\} \\
& \leq\left[1-\left(1-\epsilon_{n}\right) \alpha_{n}(1-\rho)\right]\left\|x_{n}-x_{n-1}\right\| \\
& +\left|\sigma_{n}-\sigma_{n-1}\right|\left\|G x_{n-1}-x_{n-1}\right\| \\
& +\left|\epsilon_{n}-\epsilon_{n-1}\right|\left\|\widehat{z}_{n-1}-y_{n-1}\right\|+\left|\beta_{n}-\beta_{n-1}\right| \\
& \times\left\|x_{n-1}-z_{n-1}\right\|+\left\|S_{n} x_{n-1}-S_{n-1} x_{n-1}\right\| \\
& +M_{0}\left[\left|\alpha_{n}-\alpha_{n-1}\right|+\left|\beta_{n}-\beta_{n-1}\right|\right. \\
& \left.+\left|\gamma_{n}-\gamma_{n-1}\right|+\left|\delta_{n}-\delta_{n-1}\right|\right] \\
& \leq\left[1-\left(1-\epsilon_{n}\right) \alpha_{n}(1-\rho)\right]\left\|x_{n}-x_{n-1}\right\| \\
& +\left\|S_{n} x_{n-1}-S_{n-1} x_{n-1}\right\| \\
& +M_{1}\left[\left|\alpha_{n}-\alpha_{n-1}\right|+\left|\beta_{n}-\beta_{n-1}\right|\right. \\
& +\left|\gamma_{n}-\gamma_{n-1}\right|+\left|\delta_{n}-\delta_{n-1}\right| \\
& \left.+\left|\sigma_{n}-\sigma_{n-1}\right|+\left|\epsilon_{n}-\epsilon_{n-1}\right|\right] \text {, }
\end{aligned}
$$

where $\sup _{n \geq 1}\left\{\left\|G x_{n-1}-x_{n-1}\right\|+\left\|\widehat{z}_{n-1}-y_{n-1}\right\|+\left\|x_{n-1}-z_{n-1}\right\|+\right.$ $\left.M_{0}\right\} \leq M_{1}$ for some $M_{1}>0$. Utilizing Lemma 17, we conclude from (55), conditions (i), (ii), and (vi), and the assumption on $\left\{S_{n}\right\}$ that

$$
\lim _{n \rightarrow \infty}\left\|x_{n}-x_{n+1}\right\|=0 .
$$


Furthermore, utilizing Lemma 16, we obtain from (39) and (47) that

$$
\begin{aligned}
& \left\|x_{n+1}-p\right\|^{2} \\
& =\| \epsilon_{n}\left[\left(1-\sigma_{n}\right)\left(x_{n}-p\right)+\sigma_{n}\left(G x_{n}-p\right)\right] \\
& +\left(1-\epsilon_{n}\right)\left(y_{n}-p\right) \|^{2} \\
& \leq \epsilon_{n}\left\|\left(1-\sigma_{n}\right)\left(x_{n}-p\right)+\sigma_{n}\left(G x_{n}-p\right)\right\|^{2} \\
& +\left(1-\epsilon_{n}\right)\left\|y_{n}-p\right\|^{2}-\epsilon_{n}\left(1-\epsilon_{n}\right) \\
& \times \varphi\left(\left\|\left(1-\sigma_{n}\right)\left(x_{n}-y_{n}\right)+\sigma_{n}\left(G x_{n}-y_{n}\right)\right\|\right) \\
& \leq \epsilon_{n}\left[\left(1-\sigma_{n}\right)\left\|x_{n}-p\right\|^{2}+\sigma_{n}\left\|G x_{n}-p\right\|^{2}\right. \\
& \left.-\sigma_{n}\left(1-\sigma_{n}\right) \varphi_{1}\left(\left\|x_{n}-G x_{n}\right\|\right)\right] \\
& +\left(1-\epsilon_{n}\right)\left\|y_{n}-p\right\|^{2}-\epsilon_{n}\left(1-\epsilon_{n}\right) \\
& \times \varphi\left(\left\|\left(1-\sigma_{n}\right)\left(x_{n}-y_{n}\right)+\sigma_{n}\left(G x_{n}-y_{n}\right)\right\|\right) \\
& \leq \epsilon_{n}\left[\left(1-\sigma_{n}\right)\left\|x_{n}-p\right\|^{2}+\sigma_{n}\left\|x_{n}-p\right\|^{2}\right. \\
& \left.-\sigma_{n}\left(1-\sigma_{n}\right) \varphi_{1}\left(\left\|x_{n}-G x_{n}\right\|\right)\right] \\
& +\left(1-\epsilon_{n}\right)\left[\left\|x_{n}-p\right\|+\alpha_{n}\|f(p)-p\|\right]^{2} \\
& -\epsilon_{n}\left(1-\epsilon_{n}\right) \varphi\left(\|\left(1-\sigma_{n}\right)\left(x_{n}-y_{n}\right)\right. \\
& \left.+\sigma_{n}\left(G x_{n}-y_{n}\right) \|\right) \\
& =\epsilon_{n}\left[\left\|x_{n}-p\right\|^{2}-\sigma_{n}\left(1-\sigma_{n}\right) \varphi_{1}\left(\left\|x_{n}-G x_{n}\right\|\right)\right] \\
& +\left(1-\epsilon_{n}\right)\left[\left\|x_{n}-p\right\|^{2}+\alpha_{n}\|f(p)-p\|\right. \\
& \left.\times\left(2\left\|x_{n}-p\right\|+\alpha_{n}\|f(p)-p\|\right)\right] \\
& -\epsilon_{n}\left(1-\epsilon_{n}\right) \varphi\left(\|\left(1-\sigma_{n}\right)\left(x_{n}-y_{n}\right)\right. \\
& \left.+\sigma_{n}\left(G x_{n}-y_{n}\right) \|\right) \\
& \leq\left\|x_{n}-p\right\|^{2}-\epsilon_{n} \sigma_{n}\left(1-\sigma_{n}\right) \varphi_{1}\left(\left\|x_{n}-G x_{n}\right\|\right) \\
& +\alpha_{n}\|f(p)-p\|\left(2\left\|x_{n}-p\right\|+\alpha_{n}\|f(p)-p\|\right) \\
& -\epsilon_{n}\left(1-\epsilon_{n}\right) \varphi\left(\|\left(1-\sigma_{n}\right)\left(x_{n}-y_{n}\right)\right. \\
& \left.+\sigma_{n}\left(G x_{n}-y_{n}\right) \|\right) \text {, }
\end{aligned}
$$

which immediately yields

$$
\begin{aligned}
\epsilon_{n} \sigma_{n} & \left(1-\sigma_{n}\right) \varphi_{1}\left(\left\|x_{n}-G x_{n}\right\|\right)+\epsilon_{n}\left(1-\epsilon_{n}\right) \\
& \times \varphi\left(\left\|\left(1-\sigma_{n}\right)\left(x_{n}-y_{n}\right)+\sigma_{n}\left(G x_{n}-y_{n}\right)\right\|\right) \\
\leq & \left\|x_{n}-p\right\|^{2}-\left\|x_{n+1}-p\right\|^{2}+\alpha_{n}\|f(p)-p\| \\
& \times\left(2\left\|x_{n}-p\right\|+\alpha_{n}\|f(p)-p\|\right)
\end{aligned}
$$

$$
\begin{aligned}
\leq & \left(\left\|x_{n}-p\right\|+\left\|x_{n+1}-p\right\|\right)\left\|x_{n}-x_{n+1}\right\| \\
& +\alpha_{n}\|f(p)-p\|\left(2\left\|x_{n}-p\right\|+\alpha_{n}\|f(p)-p\|\right) .
\end{aligned}
$$

So, from (56) and conditions (ii), (v), and (vi), we get

$$
\begin{gathered}
\lim _{n \rightarrow \infty} \varphi_{1}\left(\left\|x_{n}-G x_{n}\right\|\right)=0, \\
\lim _{n \rightarrow \infty} \varphi\left(\left\|\left(1-\sigma_{n}\right)\left(x_{n}-y_{n}\right)+\sigma_{n}\left(G x_{n}-y_{n}\right)\right\|\right)=0,
\end{gathered}
$$

which together with the properties of $\varphi$ and $\varphi_{1}$ implies that

$$
\begin{gathered}
\lim _{n \rightarrow \infty}\left\|x_{n}-G x_{n}\right\|=0, \\
\lim _{n \rightarrow \infty}\left\|\left(1-\sigma_{n}\right)\left(x_{n}-y_{n}\right)+\sigma_{n}\left(G x_{n}-y_{n}\right)\right\|=0 .
\end{gathered}
$$

Note that

$$
\begin{aligned}
& \left\|x_{n}-y_{n}\right\| \\
& \quad=\left\|\left(1-\sigma_{n}\right)\left(x_{n}-y_{n}\right)+\sigma_{n}\left(G x_{n}-y_{n}\right)+\sigma_{n}\left(x_{n}-G x_{n}\right)\right\| \\
& \quad \leq\left\|\left(1-\sigma_{n}\right)\left(x_{n}-y_{n}\right)+\sigma_{n}\left(G x_{n}-y_{n}\right)\right\|+\sigma_{n}\left\|x_{n}-G x_{n}\right\| \\
& \quad \leq\left\|\left(1-\sigma_{n}\right)\left(x_{n}-y_{n}\right)+\sigma_{n}\left(G x_{n}-y_{n}\right)\right\|+\left\|x_{n}-G x_{n}\right\| .
\end{aligned}
$$

Hence, from (60), it follows that

$$
\lim _{n \rightarrow \infty}\left\|x_{n}-y_{n}\right\|=0
$$

Let us show that $\lim _{n \rightarrow \infty}\left\|x_{n}-B x_{n}\right\|=0$ and $\lim _{n \rightarrow \infty} \| x_{n}-$ $S x_{n} \|=0$.

Indeed, from the definition of $y_{n}$, we can rewrite $y_{n}$ by

$$
\begin{aligned}
y_{n} & =\alpha_{n} f\left(x_{n}\right)+\beta_{n} x_{n}+\gamma_{n} B x_{n}+\delta_{n} S_{n} x_{n} \\
& =\alpha_{n} f\left(x_{n}\right)+\beta_{n} x_{n}+\left(\gamma_{n}+\delta_{n}\right) \frac{\gamma_{n} B x_{n}+\delta_{n} S_{n} x_{n}}{\gamma_{n}+\delta_{n}} \\
& =\alpha_{n} f\left(x_{n}\right)+\beta_{n} x_{n}+e_{n} z_{n}^{\prime},
\end{aligned}
$$

where $e_{n}=\gamma_{n}+\delta_{n}$ and $z_{n}^{\prime}=\left(\gamma_{n} B x_{n}+\delta_{n} S_{n} x_{n}\right) /\left(\gamma_{n}+\delta_{n}\right)$.

Utilizing Lemma 12, from (63) we have

$$
\begin{aligned}
& \left\|y_{n}-p\right\|^{2} \\
& \quad=\left\|\alpha_{n}\left(f\left(x_{n}\right)-p\right)+\beta_{n}\left(x_{n}-p\right)+e_{n}\left(z_{n}^{\prime}-p\right)\right\|^{2}
\end{aligned}
$$




$$
\begin{aligned}
\leq & \alpha_{n}\left\|f\left(x_{n}\right)-p\right\|^{2}+\beta_{n}\left\|x_{n}-p\right\|^{2} \\
& +e_{n}\left\|z_{n}^{\prime}-p\right\|^{2}-\beta_{n} e_{n} \varphi_{2}\left(\left\|z_{n}^{\prime}-x_{n}\right\|\right) \\
= & \alpha_{n}\left\|f\left(x_{n}\right)-p\right\|^{2}+\beta_{n}\left\|x_{n}-p\right\|^{2} \\
& -\beta_{n} e_{n} \varphi_{2}\left(\left\|z_{n}^{\prime}-x_{n}\right\|\right)+e_{n}\left\|\frac{\gamma_{n} B x_{n}+\delta_{n} S_{n} x_{n}}{\gamma_{n}+\delta_{n}}-p\right\|^{2} \\
= & \alpha_{n}\left\|f\left(x_{n}\right)-p\right\|^{2}+\beta_{n}\left\|x_{n}-p\right\|^{2}-\beta_{n} e_{n} \varphi_{2}\left(\left\|z_{n}^{\prime}-x_{n}\right\|\right) \\
& +e_{n}\left\|\left(1-\frac{\delta_{n}}{\gamma_{n}+\delta_{n}}\right)\left(B x_{n}-p\right)+\frac{\delta_{n}}{\gamma_{n}+\delta_{n}}\left(S_{n} x_{n}-p\right)\right\|^{2} \\
\leq & \left.\alpha_{n}\left\|f\left(x_{n}\right)-p\right\|^{2}+\beta_{n}\left\|x_{n}-p\right\|^{2}+\frac{\delta_{n}}{\gamma_{n}+\delta_{n}}\left\|S_{n} x_{n}-p\right\|\right)^{2} \\
& -\beta_{n} e_{n} \varphi_{2}\left(\left\|z_{n}^{\prime}-x_{n}\right\|\right)+e_{n}\left(\left(1-\frac{\delta_{n}}{\gamma_{n}+\delta_{n}}\right)\left\|B x_{n}-p\right\|\right. \\
\leq & \alpha_{n}\left\|f\left(x_{n}\right)-p\right\|^{2}+\left\|x_{n}-p\right\|^{2}-\beta_{n} e_{n} \varphi_{2}\left(\left\|z_{n}^{\prime}-x_{n}\right\|\right), \\
\leq & \alpha_{n}\left\|f\left(x_{n}\right)-p\right\|^{2}+\beta_{n}\left\|x_{n}-p\right\|^{2} \varphi_{2}\left(\left\|z_{n}^{\prime}-x_{n}\right\|\right)+e_{n}\left\|x_{n}-p\right\|^{2} \\
& \left.\quad \beta_{n}{ }^{2}\right)
\end{aligned}
$$

which implies that

$$
\begin{aligned}
& \beta_{n} e_{n} \varphi_{2}\left(\left\|z_{n}^{\prime}-x_{n}\right\|\right) \\
& \quad \leq \alpha_{n}\left\|f\left(x_{n}\right)-p\right\|^{2}+\left\|x_{n}-p\right\|^{2}-\left\|y_{n}-p\right\|^{2} \\
& \quad \leq \alpha_{n}\left\|f\left(x_{n}\right)-p\right\|^{2}+\left(\left\|x_{n}-p\right\|+\left\|y_{n}-p\right\|\right)\left\|x_{n}-y_{n}\right\| .
\end{aligned}
$$

From (62) and conditions (ii), (iii), and (iv), we have

$$
\lim _{n \rightarrow \infty} \varphi_{2}\left(\left\|z_{n}^{\prime}-x_{n}\right\|\right)=0
$$

From the properties of $\varphi_{2}$, we have

$$
\lim _{n \rightarrow \infty}\left\|z_{n}^{\prime}-x_{n}\right\|=0
$$

By Lemma 16, we deduce from the definition of $z_{n}^{\prime}$ the following

$$
\begin{aligned}
\left\|z_{n}^{\prime}-p\right\|^{2} & \\
& =\left\|\frac{\gamma_{n} B x_{n}+\delta_{n} S_{n} x_{n}}{\gamma_{n}+\delta_{n}}-p\right\|^{2}
\end{aligned}
$$

$$
\begin{aligned}
= & \left\|\left(1-\frac{\delta_{n}}{\delta_{n}+\gamma_{n}}\right)\left(B x_{n}-p\right)+\frac{\delta_{n}}{\delta_{n}+\gamma_{n}}\left(S_{n} x_{n}-p\right)\right\|^{2} \\
\leq & \left(1-\frac{\delta_{n}}{\delta_{n}+\gamma_{n}}\right)\left\|B x_{n}-p\right\|^{2}+\frac{\delta_{n}}{\delta_{n}+\gamma_{n}}\left\|S_{n} x_{n}-p\right\|^{2} \\
& -\frac{\delta_{n}}{\delta_{n}+\gamma_{n}}\left(1-\frac{\delta_{n}}{\delta_{n}+\gamma_{n}}\right) \varphi_{3}\left(\left\|B x_{n}-S_{n} x_{n}\right\|\right) \\
\leq & \left\|x_{n}-p\right\|^{2}-\frac{\delta_{n}}{\delta_{n}+\gamma_{n}}\left(1-\frac{\delta_{n}}{\delta_{n}+\gamma_{n}}\right) \varphi_{3}\left(\left\|B x_{n}-S_{n} x_{n}\right\|\right),
\end{aligned}
$$

which implies that

$$
\begin{aligned}
& \frac{\delta_{n}}{\delta_{n}+\gamma_{n}}\left(1-\frac{\delta_{n}}{\delta_{n}+\gamma_{n}}\right) \varphi_{3}\left(\left\|B x_{n}-S_{n} x_{n}\right\|\right) \\
& \quad \leq\left\|x_{n}-p\right\|^{2}-\left\|z_{n}^{\prime}-p\right\|^{2} \\
& \quad \leq\left(\left\|x_{n}-p\right\|+\left\|z_{n}^{\prime}-p\right\|\right)\left\|x_{n}-z_{n}^{\prime}\right\| .
\end{aligned}
$$

From (67) and condition (iii), we have

$$
\lim _{n \rightarrow \infty} \varphi_{3}\left(\left\|B x_{n}-S_{n} x_{n}\right\|\right)=0
$$

From the properties of $\varphi_{3}$, we have

$$
\lim _{n \rightarrow \infty}\left\|B x_{n}-S_{n} x_{n}\right\|=0 .
$$

From the definition of $y_{n}$, we can rewrite $y_{n}$ by

$$
\begin{aligned}
y_{n} & =\alpha_{n} f\left(x_{n}\right)+\beta_{n} x_{n}+\gamma_{n} B x_{n}+\delta_{n} S_{n} x_{n} \\
& =\beta_{n} x_{n}+\gamma_{n} B x_{n}+\left(\alpha_{n}+\delta_{n}\right) \frac{\alpha_{n} f\left(x_{n}\right)+\delta_{n} S_{n} x_{n}}{\alpha_{n}+\delta_{n}} \\
& =\beta_{n} x_{n}+\gamma_{n} B x_{n}+d_{n} z_{n}^{\prime \prime},
\end{aligned}
$$

where $d_{n}=\alpha_{n}+\delta_{n}$ and $z_{n}^{\prime \prime}=\left(\alpha_{n} f\left(x_{n}\right)+\delta_{n} S_{n} x_{n}\right) /\left(\alpha_{n}+\delta_{n}\right)$.

Utilizing Lemma 12, from (72) and the convexity of $\|\cdot\|^{2}$, we have

$$
\begin{aligned}
\| y_{n} & -p \|^{2} \\
& =\left\|\beta_{n}\left(x_{n}-p\right)+\gamma_{n}\left(B x_{n}-p\right)+d_{n}\left(z_{n}^{\prime \prime}-p\right)\right\|^{2} \\
& \leq \beta_{n}\left\|x_{n}-p\right\|^{2}+\gamma_{n}\left\|B x_{n}-p\right\|^{2}
\end{aligned}
$$




$$
\begin{aligned}
& +d_{n}\left\|z_{n}^{\prime \prime}-p\right\|^{2}-\beta_{n} \gamma_{n} \varphi_{4}\left(\left\|x_{n}-B x_{n}\right\|\right) \\
= & \beta_{n}\left\|x_{n}-p\right\|^{2}+\gamma_{n}\left\|B x_{n}-p\right\|^{2} \\
& +d_{n}\left\|\frac{\alpha_{n} f\left(x_{n}\right)+\delta_{n} S_{n} x_{n}}{\alpha_{n}+\delta_{n}}-p\right\|^{2}-\beta_{n} \gamma_{n} \varphi_{4}\left(\left\|x_{n}-B x_{n}\right\|\right) \\
= & \beta_{n}\left\|x_{n}-p\right\|^{2}+\gamma_{n}\left\|B x_{n}-p\right\|^{2} \\
& +d_{n} \| \frac{\alpha_{n}}{\alpha_{n}+\delta_{n}}\left(f\left(x_{n}\right)-p\right) \\
& \quad+\left(1-\frac{\alpha_{n}}{\alpha_{n}+\delta_{n}}\right)\left(S_{n} x_{n}-p\right)\|\|^{2} \\
& -\beta_{n} \gamma_{n} \varphi_{4}\left(\left\|x_{n}-B x_{n}\right\|\right) \\
\leq & \beta_{n}\left\|x_{n}-p\right\|^{2}+\gamma_{n}\left\|B x_{n}-p\right\|^{2} \\
& +\delta_{n}\left\|x_{n}-p\right\|^{2}-\beta_{n} \gamma_{n} \varphi_{4}\left(\left\|x_{n}-B x_{n}\right\|\right) \\
& +d_{n}\left[\frac{\alpha_{n}}{\alpha_{n}+\delta_{n}}\left\|f\left(x_{n}\right)-p\right\|^{2}\right. \\
& +\beta_{n} \gamma_{n} \varphi_{4}\left(\left\|x_{n}-B x_{n}-p\right\|^{2}+x_{n}-p x_{n}\left\|B x_{n}-p\right\|^{2}+\alpha_{n}\left\|f\left(x_{n}\right)-p\right\|^{2}-\beta_{n} \gamma_{n} \varphi_{4}\left(\left\|x_{n}-B x_{n}\right\|\right), p \|^{2} \gamma_{n}\left(\left\|x_{n}-B x_{n}\right\|\right)\right. \\
& \left.+\left(1-\frac{\alpha_{n}}{\alpha_{n}+\delta_{n}}\right)\left\|S_{n} x_{n}-p\right\|^{2}\right]
\end{aligned}
$$

which implies that

$$
\begin{aligned}
& \beta_{n} \gamma_{n} \varphi_{4}\left(\left\|x_{n}-B x_{n}\right\|\right) \\
& \quad \leq\left\|x_{n}-p\right\|^{2}-\left\|y_{n}-p\right\|^{2}+\alpha_{n}\left\|f\left(x_{n}\right)-p\right\|^{2} \\
& \quad \leq\left(\left\|x_{n}-p\right\|+\left\|y_{n}-p\right\|\right)\left\|x_{n}-y_{n}\right\|+\alpha_{n}\left\|f\left(x_{n}\right)-p\right\|^{2} .
\end{aligned}
$$

From (62), (74), and conditions (ii), (iii), and (iv), we have

$$
\lim _{n \rightarrow \infty} \varphi_{4}\left(\left\|x_{n}-B x_{n}\right\|\right)=0 .
$$

By the properties of $\varphi_{4}$, we have

$$
\lim _{n \rightarrow \infty}\left\|x_{n}-B x_{n}\right\|=0 .
$$

From (71), (76), and

$$
\left\|x_{n}-S_{n} x_{n}\right\| \leq\left\|x_{n}-B x_{n}\right\|+\left\|B x_{n}-S_{n} x_{n}\right\|,
$$

we have

$$
\lim _{n \rightarrow \infty}\left\|x_{n}-S_{n} x_{n}\right\|=0
$$

Observe that

$$
\left\|x_{n}-S x_{n}\right\| \leq\left\|x_{n}-S_{n} x_{n}\right\|+\left\|S_{n} x_{n}-S x_{n}\right\|
$$

Utilizing Lemma 13, we conclude from (78) that

$$
\lim _{n \rightarrow \infty}\left\|x_{n}-S x_{n}\right\|=0
$$

Define a mapping $W x=\left(1-\theta_{1}-\theta_{2}\right) B x+\theta_{1} S x+\theta_{2} G x$, where $\theta_{1}, \theta_{2} \in(0,1)$ are two constants with $\theta_{1}+\theta_{2}<1$. Then by Lemma 15, we have $\operatorname{Fix}(W)=\operatorname{Fix}(B) \cap \operatorname{Fix}(S) \cap \operatorname{Fix}(G)=\Delta$. We observe that

$$
\begin{aligned}
\left\|x_{n}-W x_{n}\right\| & \\
= & \|\left(1-\theta_{1}-\theta_{2}\right)\left(x_{n}-B x_{n}\right) \\
\quad & +\theta_{1}\left(x_{n}-S x_{n}\right)+\theta_{2}\left(x_{n}-G x_{n}\right) \| \\
\leq & \left(1-\theta_{1}-\theta_{2}\right)\left\|x_{n}-B x_{n}\right\| \\
& +\theta_{1}\left\|x_{n}-S x_{n}\right\|+\theta_{2}\left\|x_{n}-G x_{n}\right\| .
\end{aligned}
$$

From (60), (76), and (80), we obtain

$$
\lim _{n \rightarrow \infty}\left\|x_{n}-W x_{n}\right\|=0
$$

Now, we claim that

$$
\limsup _{n \rightarrow \infty}\left\langle f(q)-q, J\left(x_{n}-q\right)\right\rangle \leq 0
$$

where $q=s-\lim _{t \rightarrow 0} x_{t}$ with $x_{t}$ being the fixed point of the contraction

$$
x \longmapsto t f(x)+(1-t) W x
$$

Then $x_{t}$ solves the fixed point equation $x_{t}=t f\left(x_{t}\right)+(1-$ $t) W x_{t}$. Thus we have

$$
x_{t}-x_{n}=(1-t)\left(W x_{t}-x_{n}\right)+t\left(f\left(x_{t}\right)-x_{n}\right) \text {. }
$$

By Lemma 9, we conclude that

$$
\begin{aligned}
\| x_{t}- & x_{n} \|^{2} \\
= & \left\|(1-t)\left(W x_{t}-x_{n}\right)+t\left(f\left(x_{t}\right)-x_{n}\right)\right\|^{2} \\
\leq & (1-t)^{2}\left\|W x_{t}-x_{n}\right\|^{2} \\
& +2 t\left\langle f\left(x_{t}\right)-x_{n}, J\left(x_{t}-x_{n}\right)\right\rangle
\end{aligned}
$$




$$
\begin{aligned}
\leq & (1-t)^{2}\left(\left\|W x_{t}-W x_{n}\right\|+\left\|W x_{n}-x_{n}\right\|\right)^{2} \\
& +2 t\left\langle f\left(x_{t}\right)-x_{n}, J\left(x_{t}-x_{n}\right)\right\rangle \\
\leq & (1-t)^{2}\left(\left\|x_{t}-x_{n}\right\|+\left\|W x_{n}-x_{n}\right\|\right)^{2} \\
& +2 t\left\langle f\left(x_{t}\right)-x_{n}, J\left(x_{t}-x_{n}\right)\right\rangle \\
= & (1-t)^{2}\left[\left\|x_{t}-x_{n}\right\|^{2}+2\left\|x_{t}-x_{n}\right\|\right. \\
& \left.\quad \quad \quad\left\|W x_{n}-x_{n}\right\|+\left\|W x_{n}-x_{n}\right\|^{2}\right] \\
& +2 t\left\langle f\left(x_{t}\right)-x_{t}, J\left(x_{t}-x_{n}\right)\right\rangle \\
& +2 t\left\langle x_{t}-x_{n}, J\left(x_{t}-x_{n}\right)\right\rangle \\
= & \left(1-2 t+t^{2}\right)\left\|x_{t}-x_{n}\right\|^{2}+f_{n}(t) \\
& +2 t\left\langle f\left(x_{t}\right)-x_{t}, J\left(x_{t}-x_{n}\right)\right\rangle+2 t\left\|x_{t}-x_{n}\right\|^{2},
\end{aligned}
$$

where

$$
\begin{aligned}
f_{n}(t)= & (1-t)^{2}\left(2\left\|x_{t}-x_{n}\right\|+\left\|x_{n}-W x_{n}\right\|\right) \\
& \times\left\|x_{n}-W x_{n}\right\| \longrightarrow 0, \quad \text { as } n \longrightarrow \infty .
\end{aligned}
$$

It follows from (86) that

$$
\left\langle x_{t}-f\left(x_{t}\right), J\left(x_{t}-x_{n}\right)\right\rangle \leq \frac{t}{2}\left\|x_{t}-x_{n}\right\|^{2}+\frac{1}{2 t} f_{n}(t) .
$$

Letting $n \rightarrow \infty$ in (88) and noticing (87), we derive

$$
\limsup _{n \rightarrow \infty}\left\langle x_{t}-f\left(x_{t}\right), J\left(x_{t}-x_{n}\right)\right\rangle \leq \frac{t}{2} M_{2},
$$

where $M_{2}>0$ is a constant such that $\left\|x_{t}-x_{n}\right\|^{2} \leq M_{2}$ for all $t \in(0,1)$ and $n \geq 0$. Taking $t \rightarrow 0$ in (89), we have

$$
\limsup _{t \rightarrow 0} \limsup _{n \rightarrow \infty}\left\langle x_{t}-f\left(x_{t}\right), J\left(x_{t}-x_{n}\right)\right\rangle \leq 0 .
$$

On the other hand, we have

$$
\begin{aligned}
\langle f(q) & \left.-q, J\left(x_{n}-q\right)\right\rangle \\
= & \left\langle f(q)-q, J\left(x_{n}-q\right)\right\rangle-\left\langle f(q)-q, J\left(x_{n}-x_{t}\right)\right\rangle \\
& +\left\langle f(q)-q, J\left(x_{n}-x_{t}\right)\right\rangle \\
& -\left\langle f(q)-x_{t}, J\left(x_{n}-x_{t}\right)\right\rangle+\left\langle f(q)-x_{t}, J\left(x_{n}-x_{t}\right)\right\rangle \\
& -\left\langle f\left(x_{t}\right)-x_{t}, J\left(x_{n}-x_{t}\right)\right\rangle \\
& +\left\langle f\left(x_{t}\right)-x_{t}, J\left(x_{n}-x_{t}\right)\right\rangle \\
= & \left\langle f(q)-q, J\left(x_{n}-q\right)-J\left(x_{n}-x_{t}\right)\right\rangle \\
& +\left\langle x_{t}-q, J\left(x_{n}-x_{t}\right)\right\rangle \\
& +\left\langle f(q)-f\left(x_{t}\right), J\left(x_{n}-x_{t}\right)\right\rangle \\
& +\left\langle f\left(x_{t}\right)-x_{t}, J\left(x_{n}-x_{t}\right)\right\rangle .
\end{aligned}
$$

It follows that

$$
\begin{aligned}
\limsup _{n \rightarrow \infty}\left\langle f(q)-q, J\left(x_{n}-q\right)\right\rangle \\
\leq \limsup _{n \rightarrow \infty}\left\langle f(q)-q, J\left(x_{n}-q\right)-J\left(x_{n}-x_{t}\right)\right\rangle \\
\quad+\left\|x_{t}-q\right\| \limsup _{n \rightarrow \infty}\left\|x_{n}-x_{t}\right\| \\
\quad+\rho\left\|q-x_{t}\right\| \limsup _{n \rightarrow \infty}\left\|x_{n}-x_{t}\right\| \\
\quad+\limsup _{n \rightarrow \infty}\left\langle f\left(x_{t}\right)-x_{t}, J\left(x_{n}-x_{t}\right)\right\rangle .
\end{aligned}
$$

Taking into account that $x_{t} \rightarrow q$ as $t \rightarrow 0$, we have

$$
\begin{aligned}
& \limsup _{n \rightarrow \infty}\left\langle f(q)-q, J\left(x_{n}-q\right)\right\rangle \\
& \quad=\limsup _{t \rightarrow 0} \limsup _{n \rightarrow \infty}\left\langle f(q)-q, J\left(x_{n}-q\right)\right\rangle \\
& \quad \leq \lim \sup \limsup _{n \rightarrow \infty}\left\langle f(q)-q, J\left(x_{n}-q\right)-J\left(x_{n}-x_{t}\right)\right\rangle .
\end{aligned}
$$

Since $X$ has a uniformly Fréchet differentiable norm, the duality mapping $J$ is norm-to-norm uniformly continuous on bounded subsets of $X$. Consequently, the two limits are interchangeable and hence (83) holds. Noticing that $J$ is norm-to-norm uniformly continuous on bounded subsets of $X$, we deduce from (62) that

$$
\begin{aligned}
& \limsup _{n \rightarrow \infty}\left\langle f(q)-q, J\left(y_{n}-q\right)\right\rangle \\
&=\limsup _{n \rightarrow \infty}\left(\left\langlef(q)-q, J\left(x_{n}-q\right)\right.\right.\left.\quad+\left\langle f(q)-q, J\left(y_{n}-q\right)-J\left(x_{n}-q\right)\right\rangle\right) \\
& \\
&=\limsup _{n \rightarrow \infty}\left\langle f(q)-q, J\left(x_{n}-q\right)\right\rangle \leq 0 .
\end{aligned}
$$

Finally, let us show that $x_{n} \rightarrow q$ as $n \rightarrow \infty$. Indeed, utilizing Lemma 9, we obtain from (47) that

$$
\begin{aligned}
& \left\|y_{n}-q\right\|^{2} \\
& =\| \alpha_{n}\left(f\left(x_{n}\right)-f(q)\right)+\beta_{n}\left(x_{n}-q\right)+\gamma_{n}\left(B x_{n}-q\right) \\
& \quad+\delta_{n}\left(S_{n} x_{n}-q\right)+\alpha_{n}(f(q)-q) \|^{2} \\
& \leq \| \alpha_{n}\left(f\left(x_{n}\right)-f(q)\right)+\beta_{n}\left(x_{n}-q\right) \\
& \quad+\gamma_{n}\left(B x_{n}-q\right)+\delta_{n}\left(S_{n} x_{n}-q\right) \|^{2} \\
& +2 \alpha_{n}\left\langle f(q)-q, J\left(y_{n}-q\right)\right\rangle \\
& \leq \alpha_{n}\left\|f\left(x_{n}\right)-f(q)\right\|^{2}+\beta_{n}\left\|x_{n}-q\right\|^{2} \\
& + \\
& +\gamma_{n}\left\|B x_{n}-q\right\|^{2}+\delta_{n}\left\|S_{n} x_{n}-q\right\|^{2} \\
& +
\end{aligned}
$$




$$
\begin{aligned}
\leq & \alpha_{n} \rho\left\|x_{n}-q\right\|^{2}+\beta_{n}\left\|x_{n}-q\right\|^{2}+\gamma_{n}\left\|x_{n}-q\right\| \\
& +\delta_{n}\left\|x_{n}-q\right\|^{2}+2 \alpha_{n}\left\langle f(q)-q, J\left(y_{n}-q\right)\right\rangle \\
= & \left(1-\alpha_{n}(1-\rho)\right)\left\|x_{n}-q\right\|^{2} \\
& +2 \alpha_{n}\left\langle f(q)-q, J\left(y_{n}-q\right)\right\rangle,
\end{aligned}
$$

and hence

$$
\begin{aligned}
& \left\|x_{n+1} q\right\|^{2} \\
& =\| \epsilon_{n}\left[\left(1-\sigma_{n}\right)\left(x_{n}-q\right)+\sigma_{n}\left(G x_{n}-q\right)\right] \\
& +\left(1-\epsilon_{n}\right)\left(y_{n}-q\right) \|^{2} \\
& \leq \epsilon_{n}\left\|\left(1-\sigma_{n}\right)\left(x_{n}-q\right)+\sigma_{n}\left(G x_{n}-q\right)\right\|^{2} \\
& +\left(1-\epsilon_{n}\right)\left\|y_{n}-q\right\|^{2} \\
& \leq \epsilon_{n}\left[\left(1-\sigma_{n}\right)\left\|x_{n}-q\right\|^{2}\right. \\
& \left.+\sigma_{n}\left\|G x_{n}-q\right\|^{2}\right]+\left(1-\epsilon_{n}\right)\left\|y_{n}-q\right\|^{2} \\
& \leq \epsilon_{n}\left[\left(1-\sigma_{n}\right)\left\|x_{n}-q\right\|^{2}+\sigma_{n}\left\|x_{n}-q\right\|^{2}\right] \\
& +\left(1-\epsilon_{n}\right)\left\|y_{n}-q\right\|^{2} \\
& =\epsilon_{n}\left\|x_{n}-q\right\|^{2}+\left(1-\epsilon_{n}\right)\left\|y_{n}-q\right\|^{2} \\
& \leq \epsilon_{n}\left\|x_{n}-q\right\|^{2}+\left(1-\epsilon_{n}\right) \\
& \times\left[\left(1-\alpha_{n}(1-\rho)\right)\left\|x_{n}-q\right\|^{2}\right. \\
& \left.+2 \alpha_{n}\left\langle f(q)-q, J\left(y_{n}-q\right)\right\rangle\right] \\
& =\left[1-\left(1-\epsilon_{n}\right) \alpha_{n}(1-\rho)\right]\left\|x_{n}-q\right\|^{2} \\
& +2\left(1-\epsilon_{n}\right) \alpha_{n}\left\langle f(q)-q, J\left(y_{n}-q\right)\right\rangle \\
& =\left[1-\left(1-\epsilon_{n}\right) \alpha_{n}(1-\rho)\right]\left\|x_{n}-q\right\|^{2} \\
& +\left(1-\epsilon_{n}\right) \alpha_{n}(1-\rho) \frac{2\left\langle f(q)-q, J\left(y_{n}-q\right)\right\rangle}{1-\rho} \text {. }
\end{aligned}
$$

Applying Lemma 8 to (96), we conclude from conditions (ii) and (vi) and (94) that $x_{n} \rightarrow q$ as $n \rightarrow \infty$. This completes the proof.

Corollary 19. Let $X$ be a uniformly convex and 2-uniformly smooth Banach space and let $C$ be a nonempty closed convex subset of $X$ such that $C \pm C \subset C$. Let $\Pi_{C}$ be a sunny nonexpansive retraction from $X$ onto $C$. Let $T, F: X \rightarrow$ $C B(X)$, and $A: C \rightarrow 2^{C}$ be three multivalued mappings, let $g: X \rightarrow C$ be a single-valued mapping, and let $N(\cdot, \cdot)$ : $X \times X \rightarrow C$ be a single-valued continuous mapping satisfying conditions (C1)-(C5) in Theorem 4 and (C6) $N(T x, F x)+$
$A(g(x)): X \rightarrow 2^{C} \backslash\{\emptyset\}$ is $\zeta$-inverse strongly accretive with $\zeta \geq \kappa^{2}$.

Let $T_{i}: C \rightarrow X$ be a $\eta_{i}$-strictly pseudocontractive mapping for each $i=1, \ldots, N$. Define the mapping $G_{i}: C \rightarrow$ $C$ by $G_{i}=\Pi_{C}\left(I-\lambda_{i}\left(I-T_{i}\right)\right)$ for $i=1, \ldots, N$, where $\lambda_{i} \in$ $\left(0, \eta_{i} / \kappa^{2}\right)$, and $\kappa$ is the 2 -uniformly smooth constant of $X$. Let $B: C \rightarrow C$ be the $K$-mapping generated by $G_{1}, \ldots, G_{N}$ and $\rho_{1}, \ldots, \rho_{N}$, where $\rho_{i} \in(0,1)$, for all $i=1, \ldots, N-1$ and $\rho_{N} \in(0,1]$. Let $f: C \rightarrow C$ be a contraction with coefficient $\rho \in(0,1)$. Let $\left\{S_{i}\right\}_{i=0}^{\infty}$ be a countable family of nonexpansive mappings of $C$ into itself such that $\Delta:=\bigcap_{i=0}^{\infty} \operatorname{Fix}\left(S_{i}\right) \cap \Gamma \cap$ $\left(\cap_{i=1}^{N} \operatorname{Fix}\left(T_{i}\right)\right) \neq \emptyset$. Suppose that $\left\{\alpha_{n}\right\},\left\{\beta_{n}\right\},\left\{\gamma_{n}\right\},\left\{\delta_{n}\right\},\left\{\sigma_{n}\right\}$, and $\left\{\epsilon_{n}\right\}$ are the sequences in $[0,1], \alpha_{n}+\beta_{n}+\gamma_{n}+\delta_{n}=1$ and satisfy the following conditions:

(i) $\sum_{n=1}^{\infty}\left(\left|\alpha_{n}-\alpha_{n-1}\right|+\left|\beta_{n}-\beta_{n-1}\right|+\left|\gamma_{n}-\gamma_{n-1}\right|+\left|\delta_{n}-\delta_{n-1}\right|+\right.$ $\left.\left|\sigma_{n}-\sigma_{n-1}\right|+\left|\epsilon_{n}-\epsilon_{n-1}\right|\right)<\infty$;

(ii) $\lim _{n \rightarrow \infty} \alpha_{n}=0$ and $\sum_{n=0}^{\infty} \alpha_{n}=\infty$;

(iii) $\left\{\gamma_{n}\right\},\left\{\delta_{n}\right\} \subset[c, d]$ for some $c, d \in(0,1)$;

(iv) $0<\liminf _{n \rightarrow \infty} \beta_{n} \leq \lim \sup _{n \rightarrow \infty} \beta_{n}<1$;

(v) $0<\liminf _{n \rightarrow \infty} \sigma_{n} \leq \lim \sup _{n \rightarrow \infty} \sigma_{n}<1$;

(vi) $0<\liminf _{n \rightarrow \infty} \epsilon_{n} \leq \lim \sup _{n \rightarrow \infty} \epsilon_{n}<1$.

For arbitrary $x_{0} \in C$, define the sequence $\left\{x_{n}\right\}$ iteratively by

$$
\begin{aligned}
y_{n}= & \alpha_{n} f\left(x_{n}\right)+\beta_{n} x_{n}+\gamma_{n} B x_{n}+\delta_{n} S_{n} x_{n}, \\
x_{n+1}= & \epsilon_{n}\left[x_{n}-\sigma_{n}\left(N\left(w_{n}, k_{n}\right)+u_{n}\right)\right] \\
& +\left(1-\epsilon_{n}\right) y_{n}, \quad u_{n} \in A\left(g\left(x_{n}\right)\right), \quad \forall n \geq 0,
\end{aligned}
$$

where $\left\{u_{n}\right\}$ is defined by

$$
\begin{array}{r}
\left\|u_{n}-u_{n+1}\right\| \leq(1+\varepsilon) H\left(A\left(g\left(x_{n+1}\right)\right), A\left(g\left(x_{n}\right)\right)\right), \\
\forall n \geq 0,
\end{array}
$$

for any $w_{n} \in T x_{n}, k_{n} \in F x_{n}$ and some $\varepsilon>0$. Assume that $\sum_{n=0}^{\infty} \sup _{x \in D}\left\|S_{n+1} x-S_{n} x\right\|<\infty$ for any bounded subset $D$ of $C$ and let $S$ be a mapping of $C$ into itself defined by $S x=\lim _{n \rightarrow \infty} S_{n} x$ for all $x \in C$ and suppose that $\operatorname{Fix}(S)=$ $\bigcap_{i=0}^{\infty} \operatorname{Fix}\left(S_{i}\right)$. Then $\left\{x_{n}\right\}$ converges strongly to $q \in \Delta$, which solves the following VIP:

$$
\langle q-f(q), J(q-p)\rangle \leq 0, \quad \forall p \in \Delta
$$

and, for any $w \in T q$ and $k \in F q,(q, w, k)$ is a solution of the MVVI (16).

Proof. Since $T_{i}$ is a $\eta_{i}$-strictly pseudocontractive mapping for each $i=1, \ldots, N$, it is known that $A_{i}:=I-T_{i}$ is $\eta_{i}$-inverse strongly accretive for each $i=1, \ldots, N$. In Theorem 18, we put $G_{i}=\Pi_{C}\left(I-\lambda_{\mathrm{i}} A_{i}\right)$ for $i=1, \ldots, N$, where $\lambda_{i} \in\left(0, \eta_{i} / \kappa^{2}\right)$. 
It is not hard to see that $\operatorname{Fix}\left(T_{i}\right)=\operatorname{VI}\left(C, A_{i}\right)$. As a matter of fact, we have, for $\lambda_{i}>0$,

$$
\begin{aligned}
u \in & \operatorname{VI}\left(C, A_{i}\right) \\
& \Longleftrightarrow\left\langle A_{i} u, J(y-u)\right\rangle \geq 0 \quad \forall y \in C \\
& \Longleftrightarrow\left\langle u-\lambda_{i} A_{i} u-u, J(u-y)\right\rangle \geq 0 \quad \forall y \in C \\
& \Longleftrightarrow u=\Pi_{C}\left(u-\lambda_{i} A_{i} u\right) \\
& \Longleftrightarrow u=\Pi_{C}\left(u-\lambda_{i} u+\lambda_{i} T_{i} u\right) \\
& \Longleftrightarrow\left\langle u-\lambda_{i} u+\lambda_{i} T_{i} u-u, J(u-y)\right\rangle \geq 0 \quad \forall y \in C \\
& \Longleftrightarrow\left\langle u-T_{i} u, J(u-y)\right\rangle \leq 0 \quad \forall y \in C \\
& \Longleftrightarrow u=T_{i} u \\
& \Longleftrightarrow u \in \operatorname{Fix}\left(T_{i}\right) .
\end{aligned}
$$

Accordingly, we conclude that $\Delta:=\bigcap_{i=0}^{\infty} \operatorname{Fix}\left(S_{i}\right) \cap \Gamma \cap$ $\left(\cap_{i=1}^{N} \operatorname{VI}\left(C, A_{i}\right)\right)=\bigcap_{i=0}^{\infty} \operatorname{Fix}\left(S_{i}\right) \cap \Gamma \cap\left(\cap_{i=1}^{N} \operatorname{Fix}\left(T_{i}\right)\right)$. Therefore, the desired result follows from Theorem 18.

Remark 20. Theorem 18 improves, extends, supplements, and develops [5, Theorem 3.2] and [25, Theorem 3.1] in the following aspects.

(i) Kangtunyakarn's problem of finding a point of $\operatorname{Fix}(S) \cap$ $\operatorname{Fix}(V) \cap\left(\cap_{i=1}^{N} \operatorname{VI}\left(C, A_{i}\right)\right)$ (see [25, Theorem 1.1]) is extended to develop our problem of finding a point of $\bigcap_{i=0}^{\infty} \operatorname{Fix}\left(S_{i}\right) \cap \Gamma \cap$ $\left(\cap_{i=1}^{N} \mathrm{VI}\left(C, A_{i}\right)\right)$ in Theorem 18 because $B_{A}:=S((1-\alpha) I+\alpha V)$ is nonexpansive with $\alpha \in\left(0, \eta / \kappa^{2}\right)$ and $\operatorname{Fix}\left(B_{A}\right)=\operatorname{Fix}(S) \cap$ $\operatorname{Fix}(V)$ (see [25, Lemma 2.12]). It is clear that the problem of finding a point of $\bigcap_{i=0}^{\infty} \operatorname{Fix}\left(S_{i}\right) \cap \Gamma \cap\left(\cap_{i=1}^{N} \operatorname{VI}\left(C, A_{i}\right)\right)$ in Theorem 18 is more general and more subtle than the problem of finding a point of $\Gamma$ in [5, Theorem 3.2].

(ii) The iterative scheme in [25, Theorem 3.1] is extended to develop the iterative scheme (35) of Theorem 18 by virtue of the iterative schemes of [5, Theorem 3.2]. The iterative scheme (35) of Theorem 18 is more advantageous and more flexible than the iterative scheme of [10, Theorem 3.2] because it can be applied to solving three problems (i.e., MVVI (16), a finite family of VIPs, and the fixed point problem of $\left.\left\{S_{n}\right\}\right)$ and involves several parameter sequences $\left\{\alpha_{n}\right\},\left\{\beta_{n}\right\},\left\{\gamma_{n}\right\},\left\{\delta_{n}\right\}$, $\left\{\sigma_{n}\right\}$, and $\left\{\epsilon_{n}\right\}$.

(iii) Theorem 18 extends and generalizes [5, Theorems 3.2] to the setting of a countable family of nonexpansive mappings and a finite family of VIPs. In the meantime, Theorem 18 extends and generalizes Kangtunyakarn [25, Theorem 3.1] to the setting of the MVVI (16).

(iv) The iterative scheme (35) in Theorem 18 is very different from every one in [5, Theorem 3.2] and [25, Theorem 3.1] because every iterative scheme in [25, Theorem 3.1] and [5, Theorem 3.2] is one-step iterative scheme and the iterative scheme (35) in Theorem 18 is the combination of two iterative schemes in [25, Theorem 3.1] and [5, Theorem 3.2]. (v) No boundedness condition on the ranges $R(I-$ $N(T(\cdot), F(\cdot)))$ and $R(A(g(\cdot)))$ is imposed in Theorems 18 .

\section{Mann-Type Viscosity Algorithms in a Uniformly Convex Banach Space Having a Uniformly Gáteaux Differentiable Norm}

In this section, we introduce Mann-type viscosity iterative algorithms in a uniformly convex Banach space having a uniformly Gáteaux differentiable norm and show strong convergence theorems. First, we give the following useful lemma.

Lemma 21. Let $C$ be a nonempty closed convex subset of a smooth Banach space $X$ and let $A: C \rightarrow X$ be a $\xi$-strictly pseudocontractive and $\nu$-strongly accretive mapping with $\xi+$ $\nu \geq 1$. Then, for $\lambda \in(0,1]$, one has

$$
\begin{aligned}
& \|(I-\lambda A) x-(I-\lambda A) y\| \\
& \quad \leq\left\{\sqrt{\frac{1-\nu}{\xi}}+(1-\lambda)\left(1+\frac{1}{\xi}\right)\right\}\|x-y\|, \quad \forall x, y \in C .
\end{aligned}
$$

In particular, if $1-(\xi /(1+\xi))(1-\sqrt{(1-\nu) / \xi}) \leq \lambda \leq 1$, then $I-\lambda A$ is nonexpansive.

Theorem 22. Let $X$ be a nonempty closed convex subset of a uniformly convex Banach space which has a uniformly Gáteaux differentiable norm and let $C$ be a nonempty closed convex subset of $X$ such that $C \pm C \subset C$. Let $\Pi_{C}$ be a sunny nonexpansive retraction from $X$ onto $C$. Let $T, F: X \rightarrow$ $C B(X)$, and $A: C \rightarrow 2^{C}$ be three multivalued mappings, let $g: X \rightarrow C$ be a single-valued mapping, and let $N(\cdot, \cdot)$ : $X \times X \rightarrow C$ be a single-valued continuous mapping satisfying conditions (C1)-(C5) in Theorem 4. Consider that

(H6) $N(T x, F x)+A(g(x)): X \rightarrow C$ is $\xi_{0}$-strictly pseudocontractive and $\nu_{0}$-strongly accretive with $\xi_{0}+\nu_{0} \geq 1$.

Let $A_{i}: C \rightarrow X$ be $\xi_{i}$-strictly pseudocontractive and $\nu_{i}$-strongly accretive with $\xi_{i}+v_{i} \geq 1$ for each $i=1, \ldots, N$. Define the mapping $G_{i}: C \rightarrow C$ by $G_{i}=\Pi_{C}\left(I-\lambda_{i} A_{i}\right)$ where $1-\left(\xi_{i} /\left(1+\xi_{i}\right)\right)\left(1-\sqrt{\left(1-v_{i}\right) / \xi_{i}}\right) \leq \lambda_{i} \leq 1$ for each $i=1, \ldots, N$. Let $B: C \rightarrow C$ be the $K$-mapping generated by $G_{1}, \ldots, G_{N}$ and $\rho_{1}, \ldots, \rho_{N}$, where $\rho_{i} \in(0,1)$, for all $i=1, \ldots, N-1$ and $\rho_{N} \in(0,1]$. Let $f: C \rightarrow C$ be a contraction with coefficient $\rho \in(0,1)$. Let $\left\{S_{i}\right\}_{i=0}^{\infty}$ be a countable family of nonexpansive mappings of $C$ into itself such that $\Delta:=\bigcap_{i=0}^{\infty} \operatorname{Fix}\left(S_{i}\right) \cap \Gamma \cap\left(\cap_{i=1}^{N} \operatorname{VI}\left(C, A_{i}\right)\right) \neq \emptyset$. Suppose that $\left\{\alpha_{n}\right\},\left\{\beta_{n}\right\},\left\{\gamma_{n}\right\},\left\{\delta_{n}\right\},\left\{\sigma_{n}\right\}$, and $\left\{\epsilon_{n}\right\}$ are the sequences in $[0,1], \alpha_{n}+\beta_{n}+\gamma_{n}+\delta_{n}=1$ and satisfy the following conditions:

(i) $\sum_{n=1}^{\infty}\left(\left|\alpha_{n}-\alpha_{n-1}\right|+\left|\beta_{n}-\beta_{n-1}\right|+\left|\gamma_{n}-\gamma_{n-1}\right|+\left|\delta_{n}-\delta_{n-1}\right|+\right.$ $\left.\left|\sigma_{n}-\sigma_{n-1}\right|+\left|\epsilon_{n}-\epsilon_{n-1}\right|\right)<\infty$;

(ii) $\lim _{n \rightarrow \infty} \alpha_{n}=0$ and $\sum_{n=0}^{\infty} \alpha_{n}=\infty$;

(iii) $\left\{\gamma_{n}\right\},\left\{\delta_{n}\right\} \subset[c, d]$ for some $c, d \in(0,1)$; 
(iv) $0<\liminf _{n \rightarrow \infty} \beta_{n} \leq \lim \sup _{n \rightarrow \infty} \beta_{n}<1$;

(v) $0<\liminf _{n \rightarrow \infty} \sigma_{n} \leq \lim \sup _{n \rightarrow \infty} \sigma_{n}<1$;

(vi) $0<\liminf _{n \rightarrow \infty} \epsilon_{n} \leq \lim \sup _{n \rightarrow \infty} \epsilon_{n}<1$.

For arbitrary $x_{0} \in C$, define the sequence $\left\{x_{n}\right\}$ iteratively by

$$
\begin{aligned}
y_{n}= & \alpha_{n} f\left(x_{n}\right)+\beta_{n} x_{n}+\gamma_{n} B x_{n}+\delta_{n} S_{n} x_{n}, \\
x_{n+1}= & \epsilon_{n}\left[x_{n}-\sigma_{n}\left(N\left(w_{n}, k_{n}\right)+u_{n}\right)\right] \\
& +\left(1-\epsilon_{n}\right) y_{n}, u_{n} \in A\left(g\left(x_{n}\right)\right), \quad \forall n \geq 0,
\end{aligned}
$$

where $\left\{u_{n}\right\}$ is defined by

$$
\begin{array}{r}
\left\|u_{n}-u_{n+1}\right\| \leq(1+\varepsilon) H\left(A\left(g\left(x_{n+1}\right)\right), A\left(g\left(x_{n}\right)\right)\right), \\
\forall n \geq 0,
\end{array}
$$

for any $w_{n} \in T x_{n}, k_{n} \in F x_{n}$, and some $\varepsilon>0$. Assume that $\sum_{n=0}^{\infty} \sup _{x \in D}\left\|S_{n+1} x-S_{n} x\right\|<\infty$ for any bounded subset $D$ of $C$ and let $S$ be a mapping of $C$ into itself defined by $S x=\lim _{n \rightarrow \infty} S_{n} x$ for all $x \in C$ and suppose that $\operatorname{Fix}(S)=$ $\bigcap_{i=0}^{\infty} \operatorname{Fix}\left(S_{i}\right)$. Then $\left\{x_{n}\right\}$ converges strongly to $q \in \Delta$, which solves the following VIP:

$$
\langle q-f(q), J(q-p)\rangle \leq 0, \quad \forall p \in \Delta,
$$

and, for any $w \in T q$ and $k \in F q,(q, w, k)$ is a solution of the MVVI (16).

Proof. First of all, by Lemma 21, we know that $I-\lambda_{i} A_{i}$ is a nonexpansive mapping, where $1-\left(\xi_{i} /\left(1+\xi_{i}\right)\right)(1-$ $\left.\sqrt{\left(1-v_{i}\right) / \xi_{i}}\right) \leq \lambda_{i} \leq 1$ for each $i=1, \ldots, N$. Hence, from the nonexpansivity of $\Pi_{C}$, it follows that $G_{i}$ is a nonexpansive mapping for each $i=1, \ldots, N$. Since $B: C \rightarrow C$ is the $K$-mapping generated by $G_{1}, \ldots, G_{N}$ and $\rho_{1}, \ldots, \rho_{N}$, by Lemma 3, we deduce that $\operatorname{Fix}(B)=\cap_{i=1}^{N} \operatorname{Fix}\left(G_{i}\right)$. Utilizing Lemma 10 and the definition of $G_{i}$, we get $\operatorname{Fix}\left(G_{i}\right)=\operatorname{VI}\left(C, A_{i}\right)$ for each $i=1, \ldots, N$. Thus, we have

$$
\operatorname{Fix}(B)=\bigcap_{i=1}^{N} \operatorname{Fix}\left(G_{i}\right)=\bigcap_{i=1}^{N} \operatorname{VI}\left(C, A_{i}\right)
$$

Repeating the same arguments as those in the proof of Theorem 18, we can prove that for any $v \in C, \lambda>0$, there exists a point $\tilde{x} \in C$ such that $(\tilde{x}, w, k)$ is a solution of the MVVI (15), for any $w \in T \tilde{x}$ and $k \in F \tilde{x}$. In addition, in terms of Proposition 7, we know that $V+\lambda A \circ g$ is a single-valued mapping due to the fact that $V+\lambda A \circ g$ is $\phi$-strongly accretive. Assume that $N(T x, F x)+A(g(x)): X \rightarrow C$ is $\xi_{0}$-strictly pseudocontractive and $\nu_{0}$-strongly accretive with $\xi_{0}+v_{0} \geq 1$. Then by Lemma 21, we conclude that the mapping $x \mapsto x-$ $(N(T x, F x)+\lambda A(g(x)))$ is nonexpansive.
Without loss of generality, we may assume that $v=0$ and $\lambda=1$. Let $p \in \Delta$ and let $r(\geq\|f(p)-p\| /(1-\rho))$ be sufficiently large such that $x_{0} \in \bar{B}_{r}(p)=: B$. Observe that

$$
\begin{aligned}
\left\|y_{n}-p\right\| & \\
\leq & \alpha_{n}\left\|f\left(x_{n}\right)-p\right\|+\beta_{n}\left\|x_{n}-p\right\| \\
& +\gamma_{n}\left\|B x_{n}-p\right\|+\delta_{n}\left\|S_{n} x_{n}-p\right\| \\
\leq & \alpha_{n}\left(\rho\left\|x_{n}-p\right\|+\|f(p)-p\|\right)+\beta_{n}\left\|x_{n}-p\right\| \\
& +\gamma_{n}\left\|x_{n}-p\right\|+\delta_{n}\left\|x_{n}-p\right\| \\
= & \left(1-\alpha_{n}(1-\rho)\right)\left\|x_{n}-p\right\|+\alpha_{n}\|f(p)-p\| \\
\leq & \max \left\{\left\|x_{n}-p\right\|, \frac{\|f(p)-p\|}{1-\rho}\right\} .
\end{aligned}
$$

Utilizing (106) and repeating the same arguments as those in the proof of Theorem 18, we can derive $x_{n} \in B$ for all $n \geq 0$. Hence $\left\{x_{n}\right\}$ is bounded.

Let us show that $\lim _{n \rightarrow \infty}\left\|x_{n}-x_{n+1}\right\|=0$ and $\lim _{n \rightarrow \infty}\left\|x_{n}-y_{n}\right\|=0$.

Indeed, we define $G: C \rightarrow C$ by $G x:=x-(N(T x, F x)+$ $A(g(x)))$ for all $x \in C$. Then, $G$ is a nonexpansive mapping and the iterative scheme (102) can be rewritten as follows:

$$
\begin{array}{r}
y_{n}=\alpha_{n} f\left(x_{n}\right)+\beta_{n} x_{n}+\gamma_{n} B x_{n}+\delta_{n} S_{n} x_{n}, \\
x_{n+1}=\epsilon_{n}\left[\left(1-\sigma_{n}\right) x_{n}+\sigma_{n} G x_{n}\right]+\left(1-\epsilon_{n}\right) y_{n}, \\
\forall n \geq 0 .
\end{array}
$$

Repeating the same arguments as those of (56), (60), (62), (76), and (80) in the proof of Theorem 18, we can obtain that

$$
\begin{gathered}
\lim _{n \rightarrow \infty}\left\|x_{n}-x_{n+1}\right\|=0, \quad \lim _{n \rightarrow \infty}\left\|x_{n}-y_{n}\right\|=0, \\
\lim _{n \rightarrow \infty}\left\|x_{n}-G x_{n}\right\|=0, \quad \lim _{n \rightarrow \infty}\left\|x_{n}-B x_{n}\right\|=0, \\
\lim _{n \rightarrow \infty}\left\|x_{n}-S x_{n}\right\|=0 .
\end{gathered}
$$

Define a mapping $W x=\left(1-\theta_{1}-\theta_{2}\right) B x+\theta_{1} S x+\theta_{2} G x$, where $\theta_{1}, \theta_{2} \in(0,1)$ are two constants with $\theta_{1}+\theta_{2}<1$. Then by Lemma 15, we have that $\operatorname{Fix}(W)=\operatorname{Fix}(B) \cap \operatorname{Fix}(S) \cap$ $\operatorname{Fix}(G)=\Delta$. We observe that

$$
\begin{aligned}
& \left\|x_{n}-W x_{n}\right\| \\
& =\|\left(1-\theta_{1}-\theta_{2}\right)\left(x_{n}-B x_{n}\right) \\
& +\theta_{1}\left(x_{n}-S x_{n}\right)+\theta_{2}\left(x_{n}-G x_{n}\right) \| \\
& \leq\left(1-\theta_{1}-\theta_{2}\right)\left\|x_{n}-B x_{n}\right\| \\
& +\theta_{1}\left\|x_{n}-S x_{n}\right\|+\theta_{2}\left\|x_{n}-G x_{n}\right\| .
\end{aligned}
$$

From (109), we obtain

$$
\lim _{n \rightarrow \infty}\left\|x_{n}-W x_{n}\right\|=0
$$


Now, we claim that

$$
\limsup _{n \rightarrow \infty}\left\langle f(q)-q, J\left(x_{n}-q\right)\right\rangle \leq 0,
$$

where $q=s-\lim _{t \rightarrow 0} x_{t}$ with $x_{t}$ being the fixed point of the contraction

$$
x \longmapsto t f(x)+(1-t) W x .
$$

Then $x_{t}$ solves the fixed point equation $x_{t}=t f\left(x_{t}\right)+(1-$ $t) W x_{t}$. Repeating the same arguments as those of (93) in the proof of Theorem 18, we can deduce that

$$
\begin{aligned}
& \limsup _{n \rightarrow \infty}\left\langle f(q)-q, J\left(x_{n}-q\right)\right\rangle \\
& \quad=\lim _{t \rightarrow 0} \sup _{n \rightarrow \infty} \limsup _{n \rightarrow \infty}\left\langle f(q)-q, J\left(x_{n}-q\right)\right\rangle \\
& \quad \leq \lim \sup _{t \rightarrow 0} \limsup _{n \rightarrow \infty}\left\langle f(q)-q, J\left(x_{n}-q\right)-J\left(x_{n}-x_{t}\right)\right\rangle .
\end{aligned}
$$

Since $X$ has a uniformly Gáteaux differentiable norm, the duality mapping $J$ is norm-to-weak ${ }^{*}$ uniformly continuous on bounded subsets of $X$. Consequently, the two limits are interchangeable and hence (112) holds. Noticing that $J$ is norm-to-weak ${ }^{*}$ uniformly continuous on bounded subsets of $X$, we conclude from (108) that

$$
\begin{aligned}
& \limsup _{n \rightarrow \infty}\left\langle f(q)-q, J\left(y_{n}-q\right)\right\rangle \\
&=\limsup _{n \rightarrow \infty}(\left\langle f(q)-q, J\left(x_{n}-q\right)\right\rangle \\
&\left.\quad+\left\langle f(q)-q, J\left(y_{n}-q\right)-J\left(x_{n}-q\right)\right\rangle\right) \\
&=\limsup _{n \rightarrow \infty}\left\langle f(q)-q, J\left(x_{n}-q\right)\right\rangle \leq 0 .
\end{aligned}
$$

Finally, let us show that $x_{n} \rightarrow q$ as $n \rightarrow \infty$. Indeed, repeating the same arguments as those (96) in the proof of Theorem 18, we can deduce from (107) that

$$
\begin{aligned}
\left\|x_{n+1}-q\right\|^{2} & \\
\leq & {\left[1-\left(1-\epsilon_{n}\right) \alpha_{n}(1-\rho)\right]\left\|x_{n}-q\right\|^{2} } \\
& +\left(1-\epsilon_{n}\right) \alpha_{n}(1-\rho) \frac{2\left\langle f(q)-q, J\left(y_{n}-q\right)\right\rangle}{1-\rho} .
\end{aligned}
$$

Applying Lemma 8 to (116), we infer from conditions (ii) and (vi) and (115) that $x_{n} \rightarrow q$ as $n \rightarrow \infty$. This completes the proof.

Corollary 23. Let $X$ be a uniformly convex Banach space which has a uniformly Gáteaux differentiable norm and let $C$ be a nonempty closed convex subset of $X$ such that $C \pm$ $C \subset C$. Let $\Pi_{C}$ be a sunny nonexpansive retraction from $X$ onto $C$. Let T, $F: X \rightarrow C B(X)$, and $A: C \rightarrow 2^{C}$ be three multivalued mappings, let $g: X \rightarrow C$ be a singlevalued mapping, and let $N(\cdot, \cdot): X \times X \rightarrow C$ be a singlevalued continuous mapping satisfying conditions (C1)-(C5) in Theorem 4 and (H6) $N(T x, F x)+A(g(x)): X \rightarrow C$ is $\xi_{0^{-}}$ strictly pseudocontractive and $v_{0}$-strongly accretive with $\xi_{0}+$ $v_{0} \geq 1$.
For each $i=1, \ldots, N$, let $T_{i}: C \rightarrow C$ be a self-mapping such that $I-T_{i}: C \rightarrow X$ is $\xi_{i}$-strictly pseudocontractive and $\nu_{i}$-strongly accretive with $\xi_{i}+v_{i} \geq 1$. Define the mapping $G_{i}: C \rightarrow C$ by $G_{i}=\left(1-\lambda_{i}\right) I+\lambda_{i} T_{i}$ where $1-\left(\xi_{i} /(1+\right.$ $\left.\left.\xi_{i}\right)\right)\left(1-\sqrt{\left(1-v_{i}\right) / \xi_{i}}\right) \leq \lambda_{i} \leq 1$ for each $i=1, \ldots, N$. Let $B: C \rightarrow C$ be the $K$-mapping generated by $G_{1}, \ldots, G_{N}$ and $\rho_{1}, \ldots, \rho_{N}$, where $\rho_{i} \in(0,1)$, for all $i=1, \ldots, N-1$ and $\rho_{N} \in(0,1]$. Let $f: C \rightarrow C$ be a contraction with coefficient $\rho \in(0,1)$. Let $\left\{S_{i}\right\}_{i=0}^{\infty}$ be a countable family of nonexpansive mappings of $C$ into itself such that $\Delta:=\bigcap_{i=0}^{\infty} \operatorname{Fix}\left(S_{i}\right) \cap \Gamma \cap$ $\left(\cap_{i=1}^{N} \operatorname{Fix}\left(T_{i}\right)\right) \neq \emptyset$. Suppose that $\left\{\alpha_{n}\right\},\left\{\beta_{n}\right\},\left\{\gamma_{n}\right\},\left\{\delta_{n}\right\},\left\{\sigma_{n}\right\}$, and $\left\{\epsilon_{n}\right\}$ are the sequences in $[0,1], \alpha_{n}+\beta_{n}+\gamma_{n}+\delta_{n}=1$ and satisfy the following conditions:

(i) $\sum_{n=1}^{\infty}\left(\left|\alpha_{n}-\alpha_{n-1}\right|+\left|\beta_{n}-\beta_{n-1}\right|+\left|\gamma_{n}-\gamma_{n-1}\right|+\left|\delta_{n}-\delta_{n-1}\right|+\right.$ $\left.\left|\sigma_{n}-\sigma_{n-1}\right|+\left|\epsilon_{n}-\epsilon_{n-1}\right|\right)<\infty$;

(ii) $\lim _{n \rightarrow \infty} \alpha_{n}=0$ and $\sum_{n=0}^{\infty} \alpha_{n}=\infty$;

(iii) $\left\{\gamma_{n}\right\},\left\{\delta_{n}\right\} \subset[c, d]$ for some $c, d \in(0,1)$;

(iv) $0<\liminf _{n \rightarrow \infty} \beta_{n} \leq \lim \sup _{n \rightarrow \infty} \beta_{n}<1$;

(v) $0<\liminf _{n \rightarrow \infty} \sigma_{n} \leq \lim \sup _{n \rightarrow \infty} \sigma_{n}<1$;

(vi) $0<\liminf _{n \rightarrow \infty} \epsilon_{n} \leq \limsup _{n \rightarrow \infty} \epsilon_{n}<1$.

For arbitrary $x_{0} \in C$, define the sequence $\left\{x_{n}\right\}$ iteratively by

$$
\begin{aligned}
y_{n}= & \alpha_{n} f\left(x_{n}\right)+\beta_{n} x_{n}+\gamma_{n} B x_{n}+\delta_{n} S_{n} x_{n}, \\
x_{n+1}= & \epsilon_{n}\left[x_{n}-\sigma_{n}\left(N\left(w_{n}, k_{n}\right)+u_{n}\right)\right] \\
& +\left(1-\epsilon_{n}\right) y_{n}, u_{n} \in A\left(g\left(x_{n}\right)\right), \quad \forall n \geq 0,
\end{aligned}
$$

where $\left\{u_{n}\right\}$ is defined by

$$
\begin{array}{r}
\left\|u_{n}-u_{n+1}\right\| \leq(1+\varepsilon) H\left(A\left(g\left(x_{n+1}\right)\right), A\left(g\left(x_{n}\right)\right)\right), \\
\forall n \geq 0,
\end{array}
$$

for any $w_{n} \in T x_{n}, k_{n} \in F x_{n}$, and some $\varepsilon>0$. Assume that $\sum_{n=0}^{\infty} \sup _{x \in D}\left\|S_{n+1} x-S_{n} x\right\|<\infty$ for any bounded subset $D$ of $C$ and let $S$ be a mapping of $C$ into itself defined by $S x=\lim _{n \rightarrow \infty} S_{n} x$ for all $x \in C$ and suppose that $\operatorname{Fix}(S)=$ $\bigcap_{i=0}^{\infty} \operatorname{Fix}\left(S_{i}\right)$. Then $\left\{x_{n}\right\}$ converges strongly to $q \in \Delta$, which solves the following VIP:

$$
\langle q-f(q), J(q-p)\rangle \leq 0, \quad \forall p \in \Delta
$$

and, for any $w \in T q$ and $k \in F q,(q, w, k)$ is a solution of the MVVI (16).

\section{Conflict of Interests}

The authors declare that there is no conflict of interests regarding the publication of this paper.

\section{Acknowledgments}

This paper was funded by the Deanship of Scientific Research (DSR), King Abdulaziz University, Jeddah. The authors, therefore, acknowledge with thanks DSR for the technical and financial support. This research was partially supported 
(to Lu-Chuan Ceng) by the National Science Foundation of China (11071169), the Innovation Program of Shanghai Municipal Education Commission (09ZZ133), and the Ph.D. Program Foundation of Ministry of Education of China (20123127110002). Finally, the authors thank the referees for their valuable comments and appreciation.

\section{References}

[1] K. Aoyama, H. Iiduka, and W. Takahashi, "Weak convergence of an iterative sequence for accretive operators in Banach spaces," Fixed Point Theory and Applications, vol. 2006, Article ID 35390, 13 pages, 2006.

[2] L. C. Ceng and A. Petruşel, "Krasnoselski-Mann iterations for hierarchical fixed point problems for a finite family of nonself mappings in Banach spaces," Journal of Optimization Theory and Applications, vol. 146, no. 3, pp. 617-639, 2010.

[3] L.-C. Ceng, H.-K. Xu, and J.-C. Yao, "Strong convergence of an iterative method with perturbed mappings for nonexpansive and accretive operators," Numerical Functional Analysis and Optimization, vol. 29, no. 3-4, pp. 324-345, 2008.

[4] A. Kangtunyakarn and S. Suantai, "A new mapping for finding common solutions of equilibrium problems and fixed point problems of finite family of nonexpansive mappings," Nonlinear Analysis. Theory, Methods \& Applications, vol. 71, no. 10, pp. 4448-4460, 2009.

[5] C. E. Chidume, H. Zegeye, and K. R. Kazmi, "Existence and convergence theorems for a class of multi-valued variational inclusions in Banach spaces," Nonlinear Analysis. Theory, Methods \& Applications, vol. 59, no. 5, pp. 649-656, 2004.

[6] L.-C. Ceng, Q. H. Ansari, and J.-C. Yao, "Relaxed extragradient iterative methods for variational inequalities," Applied Mathematics and Computation, vol. 218, no. 3, pp. 1112-1123, 2011.

[7] L.-C. Ceng, Q. H. Ansari, M. M. Wong, and J.-C. Yao, "Mann type hybrid extragradient method for variational inequalities, variational inclusions and fixed point problems," Fixed Point Theory, vol. 13, no. 2, pp. 403-422, 2012.

[8] L.-C. Ceng, Q. H. Ansari, N.-C. Wong, and J.-C. Yao, "An extragradient-like approximation method for variational inequalities and fixed point problems," Fixed Point Theory and Applications, vol. 2011, article 22, 2011.

[9] L.-C. Ceng, N. Hadjisavvas, and N.-C. Wong, "Strong convergence theorem by a hybrid extragradient-like approximation method for variational inequalities and fixed point problems," Journal of Global Optimization, vol. 46, no. 4, pp. 635-646, 2010.

[10] L.-C. Ceng, S.-M. Guu, and J.-C. Yao, "Finding common solutions of a variational inequality, a general system of variational inequalities, and a fixed-point problem via a hybrid extragradient method," Fixed Point Theory and Applications, vol. 2011, Article ID 626159, 22 pages, 2011.

[11] L.-C. Zeng and J.-C. Yao, "Strong convergence theorem by an extragradient method for fixed point problems and variational inequality problems," Taiwanese Journal of Mathematics, vol. 10, no. 5, pp. 1293-1303, 2006.

[12] C. E. Chidume and H. Zegeye, "Approximation methods for nonlinear operator equations," Proceedings of the American Mathematical Society, vol. 131, no. 8, pp. 2467-2478, 2003.

[13] S. S. Chang, J. K. Kim, and K. H. Kim, "On the existence and iterative approximation problems of solutions for setvalued variational inclusions in Banach spaces," Journal of Mathematical Analysis and Applications, vol. 268, no. 1, pp. 89108, 2002.
[14] X. He, "On $\varphi$-strongly accretive mappings and some set-valued variational problems," Journal of Mathematical Analysis and Applications, vol. 277, no. 2, pp. 504-511, 2003.

[15] H.-K. Xu, "Iterative algorithms for nonlinear operators," Journal of the London Mathematical Society, vol. 66, no. 1, pp. 240-256, 2002.

[16] S. Reich, "Weak convergence theorems for nonexpansive mappings in Banach spaces," Journal of Mathematical Analysis and Applications, vol. 67, no. 2, pp. 274-276, 1979.

[17] Y. J. Cho, H. Zhou, and G. Guo, "Weak and strong convergence theorems for three-step iterations with errors for asymptotically nonexpansive mappings," Computers \& Mathematics with Applications, vol. 47, no. 4-5, pp. 707-717, 2004.

[18] K. Aoyama, Y. Kimura, W. Takahashi, and M. Toyoda, "Approximation of common fixed points of a countable family of nonexpansive mappings in a Banach space," Nonlinear Analysis. Theory, Methods \& Applications, vol. 67, no. 8, pp. 2350-2360, 2007.

[19] J. S. Jung, "Iterative approaches to common fixed points of nonexpansive mappings in Banach spaces," Journal of Mathematical Analysis and Applications, vol. 302, no. 2, pp. 509-520, 2005.

[20] R. E. Bruck, Jr., "Properties of fixed-point sets of nonexpansive mappings in Banach spaces," Transactions of the American Mathematical Society, vol. 179, pp. 251-262, 1973.

[21] H. K. Xu, "Inequalities in Banach spaces with applications," Nonlinear Analysis. Theory, Methods \& Applications, vol. 16, no. 12, pp. 1127-1138, 1991.

[22] I. Cioranescu, Geometry of Banach Spaces, Duality Mappings and Nonlinear Problems, vol. 62, Kluwer Academic Publishers Group, Amsterdam, The Netherland, 1990.

[23] C. H. Morales, "Surjectivity theorems for multivalued mappings of accretive type," Commentationes Mathematicae Universitatis Carolinae, vol. 26, no. 2, pp. 397-413, 1985.

[24] S. B. Nadler Jr., "Multi-valued contraction mappings," Pacific Journal of Mathematics, vol. 30, pp. 475-488, 1969.

[25] A. Kangtunyakarn, "Iterative scheme for a nonexpansive mapping, an $\eta$-strictly pseudo-contractive mapping and variational inequality problems in a uniformly convex and 2-uniformly smooth Banach space," Fixed Point Theory and Applications, vol. 2013, article 23, 2013. 


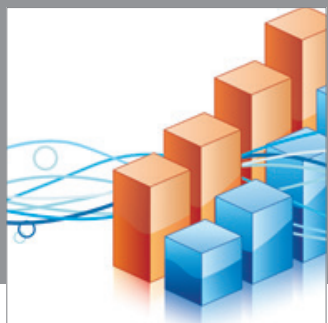

Advances in

Operations Research

mansans

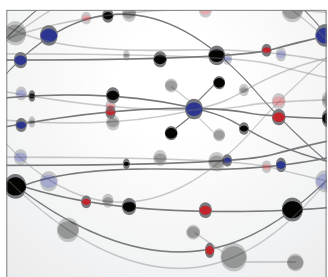

The Scientific World Journal
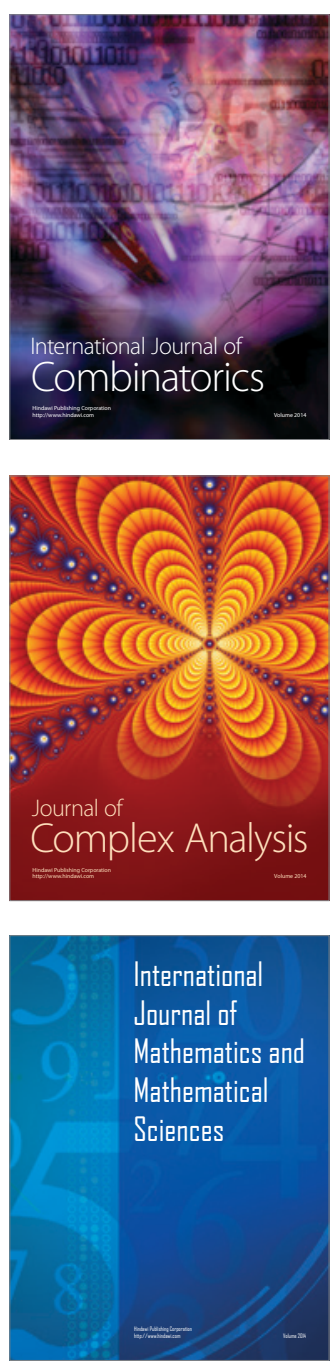
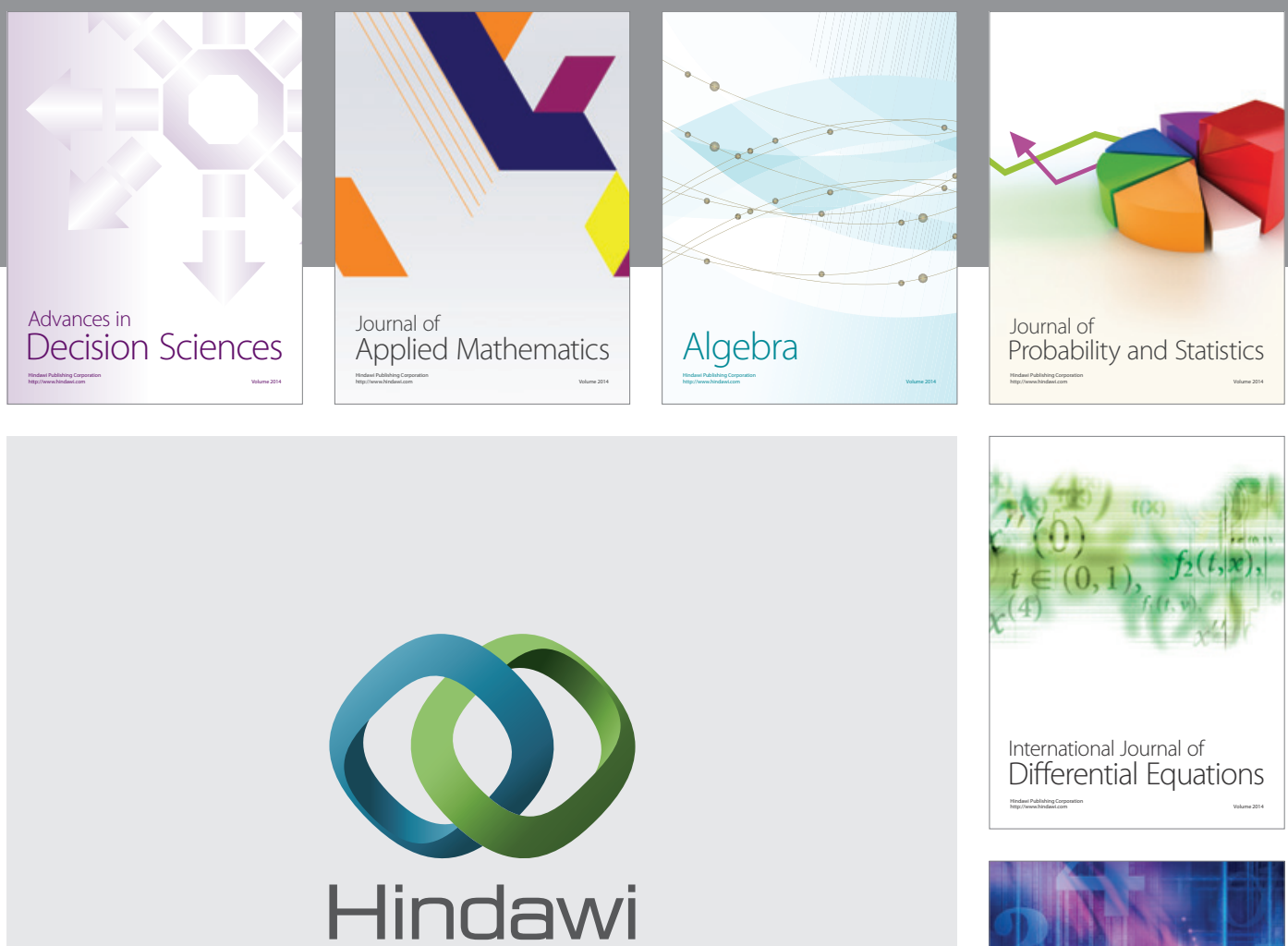

Submit your manuscripts at http://www.hindawi.com
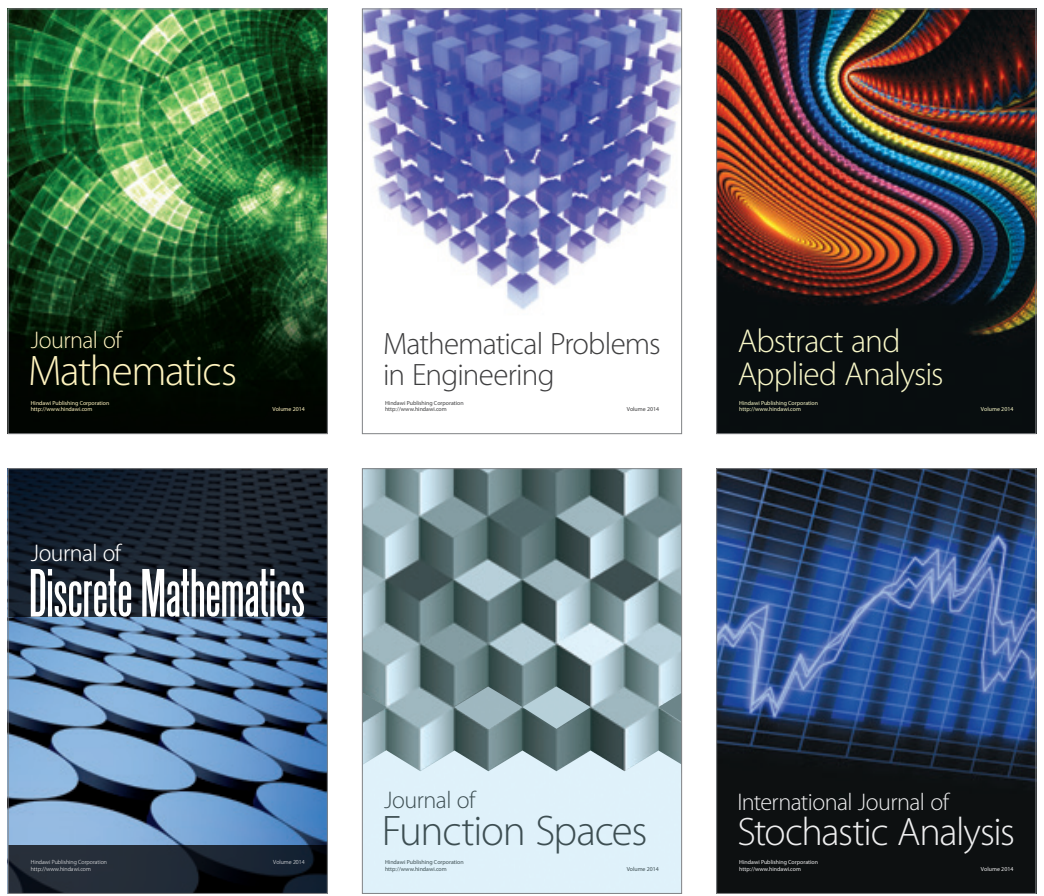

Journal of

Function Spaces

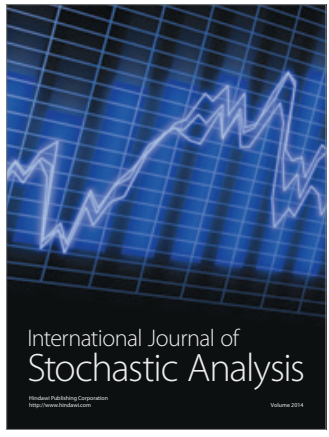

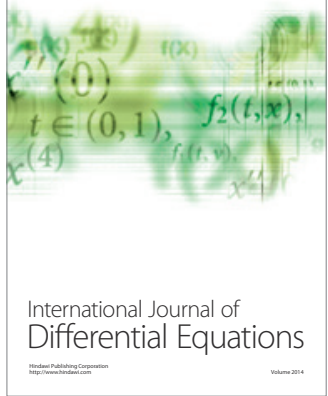
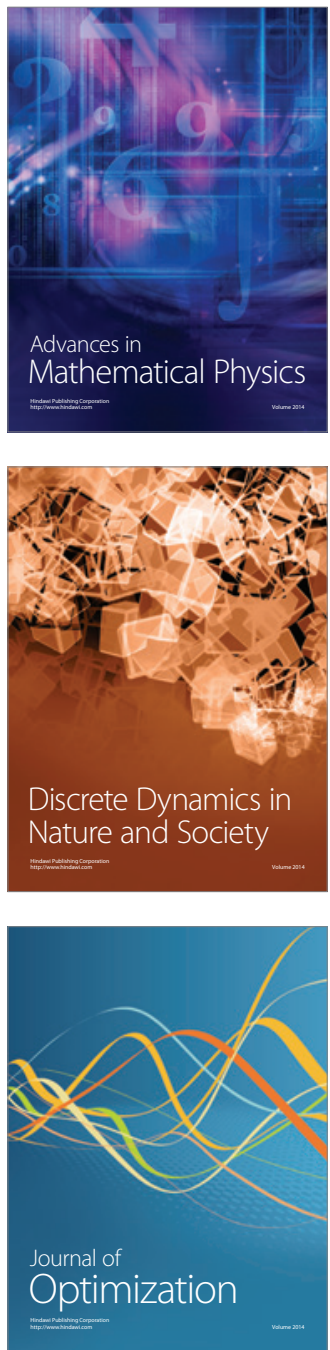